Cahiers de philosophie de l'université de

CAHIERS DE PHILOSOPHIE
DE L LUNIVERSTIE DE CAEN

Caen

$49 \mid 2012$

Levinas : au-delà du visible

\title{
Emmanuel Levinas. Textes relatifs à la soutenance de thèse du 6 juin 1961
}

Emmanuel Levinas

\section{(2) OpenEdition}

1 Journals

Édition électronique

URL : https://journals.openedition.org/cpuc/786

DOI : $10.4000 /$ cpuc.786

ISSN : 2677-6529

Éditeur

Presses universitaires de Caen

Édition imprimée

Date de publication : 31 juillet 2012

Pagination : 23-68

ISBN : 978-2-84133-410-0

ISSN : $1282-6545$

Référence électronique

Emmanuel Levinas, «Emmanuel Levinas. Textes relatifs à la soutenance de thèse du 6 juin 1961 », Cahiers de philosophie de l'université de Caen [En ligne], 49 | 2012, mis en ligne le 07 juin 2018, consulté le 31 janvier 2023. URL : http://journals.openedition.org/cpuc/786 ; DOI : https://doi.org/10.4000/ cpuc.786

\section{(c) (†) 8}

Creative Commons - Attribution - Pas d'Utilisation Commerciale 4.0 International - CC BY-NC 4.0

https://creativecommons.org/licenses/by-nc/4.0/ 


\section{LE DOSSIER DE LA SOUTENANCE DE THẼSE D'EMMANUEL LEVINAS}

\section{Monsieur Emmanuel Levinas}

Mardi

6 juin 1961

13h. 45

Salle Louis Liard
Thèse principale:

«Totalité et infini»

(exemplaire imprimé)
MM Wahl

Jankélévitch

Gabriel Marcel

$\underline{\text { Ricour }}$

Blin

«Études sur la

phénoménologie»

(deux ouvrages publiés

antérieurement)

Président des deux jurys:

M. Wahl 



\section{Emmanuel Levinas Textes relatifs à la soutenance de thèse du 6 juin $1961^{1}$}

\section{Présentation}

Le dossier de la soutenance de thèse d'Emmanuel Levinas tel qu'il se trouve dans les archives ${ }^{2}$ déposées à l'IMEC ${ }^{3}$ contient des textes préparés à l'avance, notamment l'intervention initiale d'Emmanuel Levinas pour la présentation de son travail, mais également des réponses à partir du rapport de Gabriel Marcel reçu avant la soutenance ${ }^{4}$. Il y a sans doute également des textes écrits au moment même de la soutenance. Tels qu'ils se trouvent ici restitués, ces textes ne sont pas donnés avec leurs ratures et leurs abréviations afin d'en faciliter la lecture. Il est clair que stylistiquement certains des écrits contenus dans ce dossier n'ont pas le statut d'un texte totalement rédigé et il faut y voir des notes comme support à la parole. Puisqu'il s'agit d'une soutenance de thèse, il y a un caractère responsorial des textes qui conduit parfois Emmanuel Levinas à utiliser des concepts qui ne sont pas vraiment les siens, comme «le tentateur». Tout cet ensemble constitue un témoignage important d'un grand moment de la philosophie française et est sans doute également un tournant, puisqu'il y a un avant et un après Totalité et Infini. Il offre en outre une auto-interprétation d'Emmanuel Levinas en proposant une élucidation d'une bonne partie de son lexique.

1. Textes présentés, édités et annotés par Emmanuel Housset, équipe de recherche Identité et Subjectivité.

2. Je remercie monsieur Michael Levinas de m'avoir donné l'autorisation de travailler sur ces archives et de publier le résultat de ce travail dans ce numéro des Cahiers de philosophie de l'Université de Caen. Je remercie également monsieur Olivier Corpet, directeur de l'IMEC, ainsi que madame Claire Giraudeau pour avoir favorisé ce travail qui est un élément de plus dans la coopération entre l'IMEC et l'équipe de recherche Identité et Subjectivité de l'Université de Caen Basse-Normandie.

3. Institut Mémoires de l'Édition Contemporaine, Abbaye d'Ardenne à Caen.

4. Seul ce rapport se trouve dans le dossier de la soutenance de thèse présent à l'IMEC. 


\section{Notes d'Emmanuel Levinas}

\section{Introduction}

Ce n'est pas sans un pénible embarras qu'à un certain âge on s'assoit devant le jury pour lui soumettre ses réflexions. Les précédents célèbres d'une telle situation ajoutent à ma confusion. Ils écrasent plutôt qu'ils m'encouragent. Ma gêne est d'autant plus grande que mon entreprise - qui est une entreprise de philosophie générale - peut passer pour le signe d'une naïveté que seule la jeunesse pourrait se permettre. Peut-on sérieusement après vingt-cinq siècles de philosophie s'élancer hors du commentaire des grands penseurs? Peut-on, comme en dépit de l'essence même de l'esprit, penser en dehors d'une exégèse?

En réalité la manière seule est libre dans le travail que j'ai l'honneur de vous présenter. Je n'avance rien qui ne fut déjà indiqué par la grande tradition de Platon à Bergson et, sur certains points, par une tradition encore plus ancienne.

Les maîtres de ma jeunesse dont je me dois d'évoquer les noms - hélas! par ordre de leur disparition - Henri Carteron, Charles Blondel, Maurice Halbwachs, Léon Brunschwicg, Maurice Pradines, et, pour séparer les vivants des morts, Martial Guéroult, m’ont de bonne heure initié à l'idée qu'il existe un classicisme philosophique auquel toute initiative individuelle doit se référer et dont il faut, selon le mot de Delbos, respecter le sens exact avant d'explorer le sens profond. Mais ils ont aussi enseigné la nécessité de confronter les grandes doctrines avec l'actualité de l'expérience vivante sans laquelle elles demeurent lettre morte, formule, résultat et archéologie.

Tout a été pensé, mais tout s'éclaire en dernier lieu à partir du présent - l'éternité de la vérité est peut-être dans cet accomplissement et dans cet appel à l'actualité. Selon l'image d'un exégète du deuxième siècle la parole de vérité ne se révèle vraiment que quand elle frappe comme un marteau le rocher du réel en faisant jaillir des étincelles innombrables. L'attention à la signification innombrable des phénomènes - je veux dire la phénoménologie - permet de s'ouvrir à la lumière des classiques qui, sans cela, ne brille pas de son vrai éclat. Quelles que soient les possibilités nouvelles de la phénoménologie qui nous viennent de Husserl - et je me permets d'évoquer le nom de ce maître à côté de mes maîtres français - car ils ne s'asseyent jamais en compagnie douteuse - quelles que soient les possibilités nouvelles qui nous viennent de Husserl, la phénoménologie est bien dans les traditions de notre philosophie des XVIIème et XVIIIème siècles. Elle est de Maine de Biran, de Ravaisson, de Lachelier et de Bergson. 
Les travaux que je vous présente sont donc de méthode phénoménologique dont j'aurais sans doute à répondre au cours de cette séance.

Et je voudrais maintenant exposer les problèmes que chez chacun d'entre eux j'ai été amené à aborder. J'ai bénéficié de la faveur de présenter en guise de thèse complémentaire un certain nombre d'études publiées depuis la guerre et qui portent d'une part sur le sens de la phénoménologie husserlienne et d'autre part sur le rapport entre l'existence et l'existant. La méthode phénoménologique s'appliquait à la préparation des positions qui font l'objet de ma thèse principale.

\section{De l'existence à l'existant}

I. Se place avant la relation du Même avec l'Autre. Son thème: décrire l'exister, absolument indéterminé - l'il y $a$ - où je ne distingue pas la générosité du es gibt. Montrer l'infra-structure dont surgit l'étant appelé hypostase. L'hypostase surgit comme un instant qui est le présent. Le présent n'est pas une petite portion de la durée mais la rupture avec l'il y a anonyme, pour venir à soi et surgir d'une intériorité dans un recul par rapport au point même qui se fixe comme point par ce recul même. Identification de soi avec soi où surgit le même.

Cette analyse de l'instant est menée à partir de la fatigue et, dès lors, à partir de l'effort et de l'acte. Mais l'acte premier à partir duquel l'instant se tient est tendu sur soi; concrètement: la position accomplie comme corps qui se tient sur un sol et qui est, ipso facto, conscience, car il n'est possible qu'à partir de ce retard sur soi, qui n'est pas un retard temporel, mais une intériorité (retraite, recueillement), décrite comme inconscient et comme sommeil, auxquels la conscience est adossée et où elle emprunte la distance nécessaire à sa manifestation.

II. Pourquoi préférence donnée à la fatigue et à la paresse?

- recherche de situations où se produit le clivage entre existant et existence.

- Le travail, c'est ce qui ne comporte pas d'élan ou ne peut s'y tenir. Il est une série d'arrêts. On ne peut pas le «laisser faire» tout seul. (Pour cela on invente les machines). Le travail de l'homme est dans un temps fait tout entier d'arrêts.

Toute l'entreprise de ce livre est dans la description de l'aspect nocturne de l'existence qui subsiste même dans l'hypostase: le moi - libre en tant qu'il commence en soi - est livré à soi : moi condamné à l'effort et au travail, comme à une malédiction.

La terminologie n'est pas toujours au point et ne saurait être considérée comme définitive. Par exemple: tantôt temps = bruissement anonyme de 
l'il y $a$, tantôt (mais déjà dans la perspective de la relation avec l'Autre) possibilité de renouveau et de sortie de soi, temps proprement dit.

Ce qui dans Totalité et Infini sert de point de départ - l'être séparé qui s'implante dans l'être d'une façon heureuse - se décrit déjà dans les pages 55-80 De l'existence à l'existant (sans que la distinction entre chose et élément y soit déjà faite). L'être séparé y est analysé en profondeur, mais la plus grande attention est faite à ce dépassement de l'il $y a$, à la lutte dramatique avec l'il y a (ce petit livre est bien plus dramatique que le grand) et aux divers surgissements de l'il y $a$ au sein de la position. De sorte que le thème principal de De l'existence à l'existant réside dans l'il y $a$ anonyme dominant l'hypostase. Le retour du Moi à Soi dans son identité - l'ennui de l'être séparé et l'expérience de l'irrémissibilité de l'existence - reste l'un des thèmes de Totalité et Infini et [est] abordé dans la notion de fécondité. Totalité et Infini s'achève sur ce thème dans la citation de Baudelaire.

(nouvelle fiche ${ }^{5}$ ) La substance ne peut pas se dissoudre dans la «manière d'être», dans le "comment» qu'est essentiellement le temps - durée ou temps extatique. Il faut affirmer la substance du sujet, c'est-à-dire quelque chose qui rend possible un présent autrement que dans l'unité des «extases». Ce présent ne résulte pas du fait qu'un existant subsiste sous l'écoulement du temps, ou plus exactement il ne tient pas à la nature ou à l'excellence quelconque de cet existant - mais au fait même qu'il est existant, qu'il y a de l'existant dans l'être. Le fait qu'il y a de l'existant dans l'être - c'est le fait que l'exister est à quelqu'un - qu'il y a domination sur l'anonymat de l'existant. Le présent n'est pas une petite portion de durée qui ne passe pas, mais cette domination même sur le temps. Elle n'est possible que dans le retrait par rapport à soi qu'est l'effort. Le sujet - l'existant - est la domination sur l'être. Tout mon effort consiste à sortir de l'anonymat de l'exister, vers l'existant, source de signification.

Le sujet, l'existant ou le Moi - est séparé: il repose sur le chez soi de la position où «je me tiens» corporellement (avant toute transcendance) - en soi (psychisme essentiellement) - où il retourne à soi et se pèse, d'où il s'échappera vers Autrui (Idée de l'Infini).

\section{Heidegger}

En aucune façon je ne voudrais contester son génie. On est d'ailleurs aussi ridicule en le contestant qu'en lui délivrant un satisfecit. Mais il

5. Il est difficile de dire formellement si cette fiche est la continuité de la précédente, mais c'est vraisemblable. 
représente un génie absolument hostile - non pas à moi, ce qui serait sans intérêt - mais à une tradition dont je suis l'un des témoins. Cette façon de philosopher à partir des étymologies ne me paraît pas étrangère à cause de l'incertitude de ces étymologies, mais à cause des sources auxquelles elle nous fait remonter et où, étrangement, le mystère du génie grec rejoint le mystère du génie germanique; et avec prédilection aux points où ce génie garde son irréductibilité, reste encore enfermé en lui-même et, en tout cas fermé au monde moderne où l'extension planétaire de la technique cache à Heidegger la libération de l'homme, laquelle, malgré toutes les convulsions de l'actualité, est le grand enjeu de l'actualité.

L'être de l'étant dont le resplendissement ou la dissimulation guiderait et susciterait l'étant - et l'interprétation de cet étant en métaphysique - atteste une existence fascinée par le monde, par la nature, par la calme majesté des montagnes, le mystère des forêts, l'harmonie des sentiers qui courent à travers champs, par les paysages dessinés par la pente et l'architecture des bâtiments, par la poésie, en un mot. D'où le caractère révélateur et primordial de l'art.

La nouveauté de sa vision de la nature - car c'est une vision de la nature propre à une existence enracinée et paysanne - consiste certes à avoir entrevu dans sa paix et dans son silence les articulations du langage lui-même et, par conséquent, la source même de la culture plus ancienne que l'objet - Gegenstand - en utilisant ainsi sans réserve la troisième partie des Ideen II - qui en serait l'appauvrissement et le résidu (Les étymologies heideggériennes ont ceci d'original qu'elles remontent du sens objectif et technique du mot non pas à sa désignation originelle qui serait plus matérielle et plus attachée avec (objet?) que la signification dérivée. Elles vont des significations concrètes et matérielles vers des significations infiniment plus complexes et plus riches).

Il n'en reste pas moins vrai que la philosophie de Heidegger nous ramène à un monde qui avait complètement disparu dans l'idéalisme, lequel avait absorbé ce monde dans le sujet. Contre la technique qui subordonne le monde au besoin de l'homme Heidegger affirme l'indépendance des instants qui se suffisent dans l'attention à un arbre, à un pont, entre la terre et le ciel, dans l'attente des mortels et des dieux (au pluriel). C'est du monde - ou de l'être impersonnel de l'étant qui s'y révèle - que vient toute signification.

Mon livre proteste contre cette signification de l'être. Certes, derrière les objets et avant les objets, il y a le langage de la culture - et avant lui se révèle le visage.

Dans la splendeur anonyme de la nature - où tout est au neutre - dans l'enracinement paysan ou païen qu'elle atteste, se loge la cruauté à l'égard des hommes et une indifférence éthique - l'impassibilité devant la souffrance 
des hommes dans la tendresse pour les arbres et les chiens. Dans la neutralité de l'être de l'étant, je vois l'indétermination de l'élémental.

Il me souvient de cette personne qui trouvait affreuse la guerre de 1939-45, car la cathédrale de Rouen avait été endommagée par les bombardements anglais, déformant affreusement sa ville natale et la paix de ce paysage certainement irremplaçable. Je pense que l'on peut vivre dans un monde où la cathédrale de Rouen ou même le temple de Jérusalem sont endommagés ou détruits, je pense que l'on peut vivre humainement en exil ou dans un désert où il n'y a pas d'arbres, ni ponts, ni maison. Mais je pense qu'il est difficile de vivre après les camps de la mort ou quand on a vu pendant la guerre de Corée au cinéma un enfant aux (?) errant dans les ruines et menant par la main une petite sœur qui pleure.

Qu'on ne me dise pas que je présente Heidegger dans une perspective vaine. Que l'on n'a pas le droit de situer Heidegger par rapport aux valeurs que lui seul est à même de situer, car la philosophie de Heidegger ne serait pas à situer, mais qu'elle serait par excellence situante C'est précisément cela que je conteste. Et cela par rapport à une tradition très antique, qui elle aussi est situante.

Socrate préférant la ville à la campagne parce que dans la ville on rencontre l'homme. - Presque pas de nature dans la Bible, d'où la force «Et ce fut la saison du mûrissement du raisin» ou de la colombe qui apporte une branche d'olivier dans l'Arche - Abraham plante un Tamaris à BéerShéva et le Talmud dit: (...) pas de bosquets sacrés.

Contre la prééminence heideggérienne de l'être sur l'étant, nous affirmons la priorité de l'étant. La différence - c'est l'étant échappant à l'être, autre par rapport à l'être - l'Un - quelqu'un. Cela ne signifie pas que je ne peux pas penser l'être sans penser un étant qui est. Cela signifie qu'en pensant l'être ou en comprenant l'être - j'ai déjà pris une attitude à l'égard de quelqu'un - j'ai déjà un comportement éthique - j'ai déjà adoré l'Un - je me suis reconnu au dehors. C'est l'attitude éthique qui est inconditionnellement situante.

On peut faire la psychanalyse ou l'explication marxiste de Heidegger; on peut faire l'ontologie du marxisme et de la psychanalyse, on peut faire le marxisme de la psychanalyse et de l'ontologie. On peut juger éthiquement de tout cela.

\section{Eschatologie}

La notion d'eschatologie - la fin de l'histoire - n'est pas à concevoir comme venant après mais comme à tout moment possible. La notion eschatologique de la paix n'aurait pas pu être prêchée par les prophètes si 
elle n'était pas possible à tout moment, et elle n'aurait pas pu être davantage prêchée si elle ne devait pas être à la fois annoncée comme un triomphe, comme confirmée par le monde visible (messianique) - et dans un temps assez long pour cela. La signification se dit plus vraie que les autres quand elle broie toutes les autres. Contre le désespoir du (?) il fallait donner un temps infini pour (?).

\section{Enseignement- (commandement?)}

Le visage dans sa droiture est transpercé dans son image plastique par le dire qui est l'assistance à sa propre révélation - Il a ainsi la signification - il veut dire - et par là il me vise. Le viser n'est pas le formalisme d'une prise de direction. Le mouvement de la signification - c'est le fait de m'obliger. Et cette obligation est l'ouverture de la hauteur: la maîtrise de l'Autre: je le sers - mais cette maîtrise est aussi la demande de comptes - le jugement porté par l'autre sur moi.

Servir - parce que la hauteur du Maître est aussi sa misère - il est le pauvre auquel je donne. Aux choses dont je dispose - le signifiant donne une signification, il les arrache à l'appartenance du monde à mon moi : enseignement $=$ les choses sont autre chose que ce qu'elles sont pour moi elles deviennent à tous communicables. Autrui dans ce sens - qui contient tous les autres - enseignement. Pour que le monde puisse être connu théoriquement, il faut qu'il soit au préalable arraché à ma propriété, à sa singularité - qu'il entre dans ce monde intersubjectif.

La révélation d'Autrui dans le visage est caractérisée de diverses manières:

a) le Désirable dans le Désir.

b) Le parlant (cela signifie; cela veut dire que)

c) L'enseignant

d) Le juge

e) Le Maître (que je sers)

f) Celui à qui je donne

g) Celui qui me commande

h) Présent mais pas à travers la qualité

i) L'infini

En réalité : Autrui dans le visage $=$ pas selon une qualité, mais sans masque plastique - franchise. Cette droiture $=$ parole (porte secours à soi-même $)$ signification $($ veut dire $)=$ cette parole déborde la perception 
de celui qui l'entend (enseignement) = hauteur (je donne mais à qui cela est dû $)=$ je sers $=$ mais il me juge et me commande $=$ le Désirable résume tout cela.

\section{$* * *$ \\ Visage et christianisme}

L'une de mes thèses essentielles: il y a une différence de signification entre le visage qui parle et le corps qui gesticule ou qui danse. Il y a une spiritualité de désincarnation - dans le visage, différente de l'incarnation de la signification. Spiritualisme, retour au platonisme (Cratyle: on donne des noms aux choses, sans ce sens des noms) et au monothéisme: la révélation dans le visage est dans la désincarnation et non pas dans l'incarnation.

Dans cette thèse se posent des notions de monothéisme distinctes du christianisme

1) La relation avec Dieu à travers l'homme - pas de relation directe dans l'amour qui ne traverse pas le plan éthique.

2) Le spiritualisme du visage et non pas l'incarnation. Le visage est une abstraction dans le corps, indépendamment de la culture qui est du corps.

3) Dieu n'est pas seulement étranger à toute image - mais même à toute désignation - il est le tiers qui est entre nous.

4) Le temps dominé dans l'éternité du peuple juif.

5) Le prochain inséparable du tiers.

6) Le scrupule: plus je suis juste et plus je suis coupable.

7) On n'est pas excusable - ni subjectivement ni objectivement - de ne pas nourrir celui qui a faim.

8) Pas salut de mon âme, mais mon pouvoir - de voir la larme invisible.

\section{Concrétisation page $126^{6}$}

La concrétisation dont il est question à la page 126 n'est pas seulement le passage d'une idée abstraite au contexte d'idées auquel elle fut arrachée

6. La pagination correspond à celle de la première édition de Totalité et Infini, La Haye, Martinus Nijhoff, 1961. Page 126: «La civilisation du travail et de la possession tout entière, surgit comme concrétisation de l'être séparé effectuant sa séparation". 
pour enrichir le sens de cette idée, ni le passage de l'idée à l'exemple qui l'illustre - c'est le mouvement d'une intention dont la formule abstraite comme «demeurer» ou "se représenter» ou "aborder autrui» ou «se séparer » indique la finalité, mais qui traverse tout un lacis d'intermédiaires et de circonstances - non pas en se les représentant, mais en les animant et en prenant déjà appui sur elle et en faisant corps avec eux pour aller à cette fin. En faisant corps dis-je non pas comme une partie fait corps avec un tout, mais plutôt comme une âme fait corps avec le corps qu'elle anime, un corps qui accomplit les mouvements de l'âme lesquels sont aussi parfaitement saisissables dans le mouvement en quelque sorte paralytique de la pensée. On peut dire en effet que l'union de l'âme avec le corps est la signification première (contrairement à Descartes) sur laquelle est conçu ce mouvement de concrétisation et dont la poursuite est une méthode philosophique, ou la phénoménologie elle-même.

Nous l'appelons concrétisation et non pas incarnation - car ce mouvement transitif - traverse des situations et non seulement le corps, bien que toujours à travers le corps.

La concrétisation est une méthode philosophique: en effet les diverses couches du réel qu'elle traverse révèlent une nécessité sui generis qui n'est ni logique, ni mathématique, ni dialectique. Les concepts (s'éclairent?) d'une nouvelle façon. Nous en avons partout usé dans ce livre. Je pense que l'intuition des essences, l'a priori matériel - auquel s'attachaient les premiers disciples de Husserl, a été cette méthode d'ajouter à la vision de la notion (comme contenu dans son essence) - tout ce que comportait la concrétisation de ces notions et nullement l'érection en a priori de la description du contenu empirique d'une notion.

Enfin dernière notation cette concrétisation conserve sa signification principielle, toute sa signification, puisque dans la situation ultime et quasi empirique à laquelle elle aboutit et non seulement à son niveau de condition ${ }^{7}$. La séparation suppose l'athéisme. Mais l'athéisme qui peut s'ouvrir à l'absolu de l'Autre, ne peut s'accomplir comme athéisme que si effectivement il peut se maintenir radicalement comme athéisme. De sorte que la description ne se borne pas à rechercher la corrélation ambiguë de la concrétisation empirique - mais ses mouvements ultimes peuvent se poursuivre de manière à surmonter - et en vérité $\langle$ sic $>$.

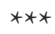

7. Ce passage est peu lisible. 


\section{L'avenir de l'élément qui est une insécurité}

C'est à proprement parler la façon particulière dont durent les choses - leur enfoncement dans une durée qui n'est pas le temps de leur mouvement - mais comme le temps de leur immobilité et qui tranche sur le temps de leur perception - temps dans lequel elles viennent comme anciennes et comme ayant leur valeur à elles et leur mystère qui fait de leur jouissance même quelque chose d'accidentel - où elle se prête seulement à nous - où elles sont capturées accidentellement. La profondeur de l'élément $=$ la durée mystérieuse du monde

\section{Le féminin}

L'objection que l'on peut me faire consiste à me reprocher

1) l'ignorance du fait que la femme est un sujet

2) de méconnaître la perspective du masculin à parler du féminin.

Je pourrais répondre à la première objection que l'idée de sujet de la représentation n'est pas spontanément dérivée et constitue déjà le résultat d'une certaine situation - être dans la maison - se représenter et que le féminin sur d'autres voies peut y aboutir également. La réciprocité se substitue à l'asymétrie du rapport interpersonnel dans l'histoire et l'économie. Le sujet n'est pas d'emblée dérivé comme (?)

Je dirai de la deuxième objection que

a) le féminin et le masculin ne sont pas seulement accomplis dans l'empirie, mais que ce sont des moments méta-empiriques. Le sujet féminin peut se vivre comme s'il était masculin et inversement, et que c'est là que réside peut-être la possibilité empirique de l'homosexualité - qu'il y a en tout cas une commutation possible sur ce terrain d'un sexe à l'autre.

b) Que cependant l'asymétrie de la relation intersubjective se répète au niveau de la sexualité et que méconnaître ce point de départ sexué c'est (rater?) la vérité.

c) Que la philosophie à partir du féminin - (peut se produire) d'un niveau plus profond que celui du sujet - mais qu'elle ne s'est pas encore (produite?) dans une civilisation essentiellement masculine et non pas neutre (quoi qu'on en dise) - et qu'il n'est pas sûr que cet abord du masculin à partir du féminin soit précisément une philosophie. C'est Diotime qui raconte à Socrate le sens de l'amour - mais c'est une vieille femme - déjà sortie de la condition féminine (à vérifier). 


\section{L'ambiguïté du corps - la conscience, pages $137-142^{8}$}

La demeure - à l'orée d'une intériorité - use.

Pour manipuler - il faut prendre $=$ posséder. Pour posséder - se trouver au bord de l'intériorité - Saisir dans la nuit de l'élément, sans être guidé par une représentation préalable - c'est découvrir un monde (antériorité de la prise sur le monde). La chose = la mobilité. La main - la demeure (la station debout) = simultanées. Donc le monde qui suppose la prise, suppose la main, suppose le corps. Le corps a été décrit jusqu'alors comme le comment de la séparation (à partir de la jouissance) - par quoi la jouissance se contente et subit une influence: se tenir et être résultat. Par la demeure s'ajourne l'influence. La demeure fait surgir la conscience. Le corps devient conscience à partir de la demeure (ce qui n'est pas dit page 139). Conscience - désincarnation grâce à la demeure. Le travail rend possible la conscience car le travail suppose la demeure. La main vient de l'intériorité - va dans le vide tâtonne.

\section{L'obscène}

Paradoxe de l'amour sexuel; paradoxe scandaleux: ambiance essentiellement impure de ce qui, par ailleurs apparaît comme l'œuvre la plus exultante de la vie - procréation d'amour.

Impureté qui n'est pas de l'ordre de l'immoralité interpersonnelle ou personnelle comme le vol ou la goinfrerie ou l'avidité.

Elle ne heurte pas simplement des préjugés: même les êtres les plus affranchis de tabous sexuels peuvent-ils s'empêcher d'éprouver la concupiscence comme sans grandeur, comme trouble, comme comportant quelque chose d'indigne de l'homme, de l'être raisonnable, comme quelque chose de par excellence matériel.

L'interprétation de cette obscénité - par la matérialité et l'animalité, n'est pas à la mesure de cette impureté. Rien n'est plus pur que la matérialité elle-même; l'obscénité sexuelle n'est pas simplement biologique. Le terme d'obscénité, que Sartre réserve parfois à la manifestation de la matière informe et absurde, est métaphorique et est au contraire emprunté précisément au sexuel.

Notre analyse de l'obscénité comme profanation - comme le phénomène initial de la profanation d'un mystère et notre description de la profanation comme la découverte de ce qui est et demeure caché dans sa découverte

8. Totalité et Infini, «Intériorité et économie», La demeure, 5. Le travail, le corps, la conscience. 
même (ce en quoi la profanation diffère du dévoilement) remonte à la situation privilégiée de l'obscène.

C'est la très grande audace de l'amour sexuel qui nous place dans la situation exceptionnelle de l'obscène.

L'originalité de l'«immoralité» obscène: elle n'entre pas simplement à sa place dans un ordre hiérarchique de valeurs ou de non valeur. On peut concevoir une hésitation de l'âme pieuse entre une valeur universelle incontestable la plus haute: sauvetage d'une vie humaine et une valeur de simple pudeur. Cf Traité (?) 9

\section{L'être est extériorité}

Je ne pense pas que le mot extériorité doive avoir un sens spatial. M. Minkovski a montré avec une pénétration particulière combien les notions spatiales impliquent de temporelles et combien les temporelles impliquent les spatiales - de sorte que le tout remonte à des situations où ces abstractions sont encore unies. J'ai cherché à fixer l'extériorité par l'altérité éthique plutôt que de décrire l'altérité par l'espace. Dire que l'être est extériorité - ce n'est pas caractériser par l'extériorité l'être de l'étant, mais une conjoncture ou l'être de l'étant ne commande précisément pas l'étant. Il ne s'agit pas ici de la subsistance d'une substance, ni d'une sortie de cette subsistance par le projet (ce qui serait l'extériorisation dont, vous avez raison, je ne veux pas), mais d'un affrontement d'un étant qui vous fait face (et par là précisément est infini). Cet être que j'affronte - n'est pas un objet, qui n'a ni face ni front. L'extériorité n'est nullement extatique. Elle vient vers nous à travers un intervalle - qui se creuse à partir de l'autre, voilà ce que j'entrevois. C'est cette façon d'être en accueillant que j'appelle obéir à l'extériorité. Je l'appelle encore religion.

La scission de l'être en multiplicité est son exercice même - et pour que cette multiplicité ne se referme aussitôt en un ensemble - il faut l'affrontement entre les multiples - il faut un visage - il faut la dimension de la Hauteur et l'infini où le visage se place. Il n'y a pas de place commune à cause de la hauteur. Et il faut le point de vue subjectif à partir duquel on peut répondre au visage. De sorte que le point de vue subjectif n'est pas un inconvénient de la révélation, mais son unique possibilité.

9. Sans doute le traité Sota du Talmud de Babylone. Je remercie Catherine Chalier de cette indication. 


\title{
Visage et langage
}

Par langage - je n'entends pas tous les produits culturels des langues et mon analyse ne s'est jamais engagée dans cette voie. Je n'aborde pas le langage dans sa structure sémantique et syntaxique - comme un système de symbolisme. Je l'aborde toujours comme une relation entre êtres qui parlent - et comme tirant de cette relation une essence qui rend seulement le symbolisme possible. Le langage n'est pas une espèce de symbolisme, le symbolisme présuppose le langage, comme la relation même de signifiance. Il s'agit là encore du langage qui précède la culture, qui a une essence éthique, qui est à sa façon universel.

\begin{abstract}
ion
On dira que cette position de la conscience est abstraite - qu'elle est considérée en dehors des conditions concrètes qui dans le développement historique rendent possible le désintéressement et la responsabilité même. Le désintéressement ne sort pas d'un conflit d'intérêt, mais suppose un idéalisme initial de quelqu'un qui renonce à ses intérêts pour magnifier l'autre. Il faut un idéalisme dont le langage est constamment parlé, même là où théoriquement le matérialisme est professé. Il faut tout l'infini d'une responsabilité pour faire seulement quelque chose.

Ma position consiste précisément à affirmer qu'une abstraction est nécessaire dans la continuité historique.

Ce qui rend possible l'utilisation même des possibilités concrètes de l'histoire - c'est l'abstraction de la bonté gratuite, c'est une position d'élu sur qui repose toute l'histoire de l'humanité. Elle seule me permet de m'insérer dans le tissu même de l'histoire et de ne pas «vivre caché» comme le voudrait l'épicurisme, de sortir de la vie privée dans la vie publique.

Abstraction - platonisme: indépendance de l'éthique par rapport à la culture.
\end{abstract}

\section{Usage légitime de l'idée de Totalité}

Je crois avoir indiqué l'usage de l'idée de totalité à partir de la synopsie de la représentation, laquelle repose, elle-même, sur l'impérialisme du Même absorbant tout Autre, réduisant les choses et les hommes à l'identité 
du Même, partant ainsi d'une conception d'après laquelle la relation entre le Même et l'Autre est hostilité, est allergique.

J'ai montré dans les pages $195-208^{10}$ comment la relation asymétrique - c'est-à-dire éthique de la relation du Même avec l'Autre, s'invertit en réciprocité à travers la guerre et la corruption - et où l'idée de totalité surgit.

L'idée de totalité semble s'imposer enfin dans la relation éthique ellemême à partir de la présence de l'humanité - des autres hommes - du tiers - dans le visage qui nous fait face. La réponse politique - à partir d'une totalité sociale - peut dès lors apparaître comme une réponse à l'appel du visage. Mais, là encore, le politique et sa totalité, dans une certaine mesure, admissible, - mais dans une certaine mesure seulement - surgit à partir de l'éthique.

«Le savoir, en tant que concret, n'existe qu'en tant que s'épanouissant concrètement en lui-même, s'englobant et se tenant dans l'unité c'està-dire en tant que totalité, et il ne peut que, par la différenciation et la détermination de ses différences, être comme la nécessité de ces différences et comme la liberté du tout ${ }^{11}$.

Pas de philosophie sans système. Un contenu ne se justifie que comme un moment du tout sinon il n'a qu'une certitude subjective. Système = conformité à un principe, mais à condition d'inclure tous les principes particuliers.

Comment une telle totalité se constitue-t-elle?

J'ai essayé cette déduction en montrant le passage du discours et de la transcendance - au monde historique où surgit l'homme - et où l'analyse est menée à la troisième personne - où la violence - guerre ou commerce - se substitue au désir et au discours - où les hommes morts laissant des œuvres s'articulant en une totalité implacable comme un destin - mais où l'homme vivant et patient conserve le pouvoir de rompre cette totalité.

\section{Sur le rapport de l'être du tout et de l'infini}

a) La distinction entre l'être et l'étant s'impose à toute pensée. La grande force de Sein und Zeit. Mais elle est perceptible avant. Principes I art. 51: Dieu n'est pas substance dans le même sens que la créature (certes il ne connaît que la différence entre nécessaire et contingent).

10. Totalité et Infini, «Le visage et l'extériorité», La relation éthique et le temps, 1. Le pluralisme et la subjectivité, début.

11. Traduction de Hegel, Encyclopédie, Introduction, $\$ 14$. 
Kant: existence n'est pas un attribut.

Bergson: la durée - ce n'est pas un étant, mais un mode d'être. Jank: la quoddité distincte de la quiddité ${ }^{12}$.

b) Contre la priorité de la connaissance de l'être (ontologie) par rapport à la relation avec l'étant $=$ éthique. Compréhension de l'être de l'étant = compréhension d'un neutre ou se fait la transmutation de l'Autre en Même, sa violation, une injustice.

c) Mais il ne s'agit pas de dire des préférences - ce ne serait pas philosophique. L'étant s'impose parce que: toute pensée qui se dirige sur l'être a déjà reconnu un étant auquel elle se dit (elle est impossible sans langage). La relation éthique est la situation originelle où la réflexion sur l'être se situe déjà. Religion = impossible de ne pas être en face de...

d) Mais l'étant n'est pas n'importe quel objet. Toute chose s'offre à la possession - qui est une prise sur l'être de son étant - lequel dans la possession est suspendu puisque l'étant possédé n'est pas en soi, mais à moi - toute chose s'offre à la prise sur son être ou à la compréhension de son être - ou à l'ontologie. L'ontologie est née de la considération du monde à posséder.

On peut se demander si l'être de l'étant dans sa neutralité n'est pas un autre nom de la matière. Et toutes choses - en dehors de ce qui se révèle dans le visage humain - se ramènent au neutre de la matière (les arbres sont du bois, les animaux sont de la viande), tout est éventuellement marchandise.

L'étant est précisément étant parce qu'il se refuse à sa dissolution dans l'anonymat de son être, et, par là, à la possession. L'étant est précisément ce qui tranche sur l'être anonyme - ce qui résiste à la neutralité. La relation éthique est cette résistance même. Elle se produit entre étants.

e) maintenant l'ontologie telle que je viens de la critiquer - n'est pas le réalisme ou une doctrine quelconque sur la réalité, mais en tant que compréhension de l'être de l'étant ou en tant que tentative de saisir la réalité en tant que intelligible dans la totalité, laquelle est impossible sans mépris pour l'altérité de l'autre.

f) Si cependant je dis que dans la guerre la force de l'être qui se révèle est la totalité, ce qui laisserait supposer un autre versant dans l'être - je ne refuserais pas à cette conséquence dans la mesure où la relation avec l'étant par excellence qu'est l'homme (la relation éthique) - ou la relation avec l'infini - n'est pas une relation avec le néant, ni ne se joue dans le néant - ou l'élévation ou la transcendance n'est pas une négativité.

12. Pour Jankélévitch? Voir V. Jankélévitch, Philosophie première, Paris, PUF, 1953. 
L'aliénation est l'expérience même de la totalité. La guerre c'est l'aliénation, la guerre et la corruption, la flatterie qui se (sert?) de la volonté avec son consentement. Pourquoi l'aliénation serait-elle mauvaise? N'est-ce pas à cause de l'exaltation du même? La morale n'est-elle pas la mise en cause du Même? Il y aurait donc deux formes de l'aliénation malgré moi. La philosophie serait-elle la préservation contre l'aliénation? La lutte contre l'aliénation, c'est précisément cela la recherche de la vérité - où l'au-dessus de l'être, ou l'idée du Bien - n'est pas une relation avec le néant.

La sortie de l'être vers le Bien - est encore une aventure réelle. Si elle n'est pas ontologie - cela veut dire qu'elle ne consiste ni à englober une totalité pour s'y dissoudre, ni à recevoir les ordres anonymes de l'être de l'étant - mais à accueillir l'infini. Je ne suis pas contre l'être, mais contre l'ontologie ou le triomphe du Même. Nous sommes dans l'être, mais être dans l'être, c'est aller au-dessus du Même - la notion de hauteur (et non pas de valeur puisqu'il s'agit d'accueillir la valeur d'un être) se produit dans l'être lui-même. Un être qui ne serait que lui-même ne serait pas.

Dira-t-on que la description de cette aventure avec le dépassement de l'ontologie et de la totalité est encore une ontologie? Mais justement le primat de l'éthique signifie que cette description ne peut jamais englober l'événement même d'où elle surgit - qu'elle est déjà dite c'est-à-dire que cet événement - en cette situation - est à nouveau une relation entre le Même et l'Autre, entre étants, une transcendance qui ne rentre pas dans la description de l'être de l'étant - que cette réflexion elle-même repose sur le langage ne peut être suscitée que par la présence d'Autrui, d'un étant.

L'être de l'étant - n'est pas dans un oubli de l'être qui s'est détourné de l'homme - il est dépassé par l'homme qui fixe l'étant. C'est son excellence.

J'emploie le terme ontologique comme adjectif formé à partir de l'óv

Fera-t-on cette différence entre liberté abstraite et concrète? L'aliénation concrète serait-elle seule en question? Philanthropie.

\section{Monade}

Quand à la page 251 je dis «L'être en tant qu'être est pour nous monade» - c'est une position contraire à la mienne que j'expose. Mon effort consiste précisément à montrer que l'exister comme tel implique multiplicité - et non pas l'unité. 


\section{Le prochain}

J'évite ce terme car il peut faire croire que la relation du Même à l'Autre repose sur la communauté du voisinage.

Ce n'est pas la proximité qui fonde le rapport entre nous, c'est la révélation du visage qui fonde la proximité et la droiture même.

Mais surtout - le prochain: c'est une préférence arbitraire, mais une préférence et comme une complicité.

Or l'un des points qui m'importent c'est le caractère public de la relation. Le tiers n'est pas absent du visage de l'autre. Il exige justice.

\section{Contradiction. Infini et expérience}

Je ne crois pas qu'il y ait contradiction entre ce que je dis page 170 sur l'expérience de l'infini et sur ce que j'en dis dans la préface. Me permettezvous de relire ce passage de la préface:

\section{Infini et Descartes}

Je ne crois pas qu'il me soit interdit de me référer à Descartes. Tout mon effort consiste à montrer que l'idée de l'infini est l'idée du Bien. Ce n'est pas la perfection - mais c'est sous une autre forme une qualification axiologique de l'infini. Description de la situation où surgit précisément l'idée de valeur inséparable de l'infinition de l'infini.

\section{Personne}

Si je n'use pas du terme de personne, c'est que la personne a un contenu culturel - est déjà un personnage. La personne d'autre part est le stade où le Moi et l'Autre sont dans l'égalité et non pas asymétrique.

\section{Face à face et éthique}

Pourquoi le face à face prendrait une valeur éthique? 
Je pense que sans cela la droiture du face à face est impossible. La signifiance éthique de l'autre ne fait qu'un avec le visage où il m'apparaît. Quand vous m'objectez la nécessité d'une rencontre pour que je puisse rencontrer un visage signifiant - vous parlez de signification dans un tout autre sens: il y aurait sur le plan éthique ou intellectuel des âmes plus intéressantes que d'autres par leur contenu que le visage exprimerait (...)

Je prends le visage comme l'ultime nudité de l'être, comme la percée de toute forme, et c'est alors que j'y distingue la signification éthique - c'est par elle que le visage parle. Celle-ci est le fait de tout être humain. C'est elle qui rend seulement la rencontre possible. Voilà pourquoi je ne fais pas intervenir la notion de rencontre. La veuve, l'orphelin - ce sont des termes concrets pour marquer cette misère du visage, l'enseignement, la maîtrise (ceux qui ne sont pas intéressants) - pour marquer sa hauteur - sauf que ces termes qui expriment la hauteur indiquent l'articulation même du langage dans des significations diverses; ce ne sont pas comme vous dites très pertinemment des individus d'un genre, mais les articulations d'une structure.

Je tiens beaucoup à ce formalisme du non formel que vous me reprochez et qui se trouve effectivement dans mon travail. Il me permet de sauver dans la décolonisation actuelle un élément permanent et certes platonicien.

Mais il faut peut-être dire deux mots de ce formalisme désontologisé de l'infini - je veux bien. Mais cela ne peut vouloir dire qu'une chose, ne pas prendre l'infini pour une chose, ne pas l'englober, s'opposer à l'être identifié avec la totalité, la particularité d'une chose ni avec l'être de l'étant. En réalité il s'agit de le placer en dehors du Même plutôt qu'en dehors de l'être.

Je répète: la relation qui consiste à dépasser l'être est un événement qui se produit dans l'être - au-dessus de l'être, n'est pas une négation de l'être. Pas la négativité. La situation est semblable à celle de l'idée du Bien chez Platon.

\section{Désir et bonté}

L'idée de l'infini m'est apparue dans son paradoxe qui consiste à penser plus qu'on ne pense - et c'est pourquoi je l'ai rapproché du désir dont l'intentionnalité peut se décrire par cette visée qui (va) au-delà de la possession de son ideatum. C'est la phénoménologie du désir qui m'a semblé déboucher sur un ideatum qui a le statut - si l'on peut s'exprimer ainsi - de l'infini. 
Désir de l'infini. Le dépassement de toute satisfaction par le désir consiste en un retournement de son intentionnalité de possession vers la générosité de la bonté. Et cependant la bonté ne se transforme pas ainsi en gratuité absolue où disparaîtrait jusqu'à la trace de la source du désir dans le moi. Nous avons cherché à surprendre la bonté à partir du désir afin de conserver à la bonté sa source subjective. - Et cela s'atteste dans le phénomène: où la bonté est comme un besoin et où l'exercice de la bonté est comme une passion. Il y a comme une souffrance pour la bonté de ne pas avoir de caresses pour se satisfaire par delà les actes.

Dans la Bible hébraique le terme qui sert à exprimer l'amour de Dieu pour ses élus (Deut 7. 7; Deut 10 ou dans le Psaume «Il m’a désiré») est le même qui désigne le désir du guerrier pour la femme captive.

Dostö̈evski.

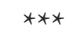

\section{Eros}

Je ne crois pas n'avoir pas entrevu la participation de la transcendance ou de la trans-substantialisation de l'eros à la jouissance du besoin. J'en parle longuement à la page 232 sous le titre de "L'ambiguïté de l'Amour» et je dis comment l'eros se donne toujours à la limite du «plus cruel et du plus égoïste des besoins». (...)

\section{Comment Dieu parle-t-il enfin!}

I Il faut toujours parler avec crainte et timidité de Dieu même en religion.

II Mon souci a consisté à approcher cet idéal à partir de l'éthique - à travers Autrui. La présence d'Autrui dans son visage - est l'ouverture même de la hauteur qui est la dimension de Dieu. «Dieu invisible - cela ne signifie pas seulement un Dieu inimaginable, mais un Dieu accessible dans la justice». L'idéal n'est pas seulement un être superlativement être, sublimation de l'objectif, ou dans une solitude amoureuse, sublimation d'un Toi» (page 51) ${ }^{13}$. C'est Platon qui a dit qu'il ne connaît d'autre hauteur que celle de l'invisible.

13. Totalité et Infini, «Le Même et l'Autre», Séparation et Discours, 6. Le métaphysique et l'humain. 
III La phénoménologie de la relation impersonnelle rencontre d'ailleurs la dimension du divin - en dehors de cette hauteur et de cette humilité qui s'ouvre dans le visage d'autrui. Elle le trouve aussi comme le Troisième qui est entre nous, entre Moi et Autrui. Car toute l'humanité - tous les tiers - sont présents dans le visage d'Autrui. Ce sont eux qui empêchent que l'accueil de l'Autre ne se transforme en complicité. Et c'est pourquoi l'accueil de l'Autre n'est pas amour, et l'Autre pas prochain. (La charité est exclusive du tiers). Le mot dialogue ne me suffit pas plus: le langage est toujours public. Mais comme la totalité des tiers n'est pas seulement une totalité qui se résume - mais ce sur quoi le langage débouche - c'est Dieu qui représente ce témoin invisible du dialogue.

Les religions où Dieu surgit dans la relation éthique me semblent donc assez nettement indiquées.

Mais l'effort de mon analyse tend surtout à montrer que - en partant de la relation avec Autrui - nous aboutissons à un Dieu qui parle - ce qui signifie: à aucun moment il ne se joue de nous, jamais en partant du Dieu éthique - nous ne nous trouvons entraînés à notre insu dans un drame sacré qui déborde nos intentions - et dans ce sens notre position est rationaliste.

Elle consiste certes à admettre une hétéronomie - et à poser la révélation comme la base de l'universalité même de la raison - mais la première parole de cette révélation est dans l'ouverture même du visage.

Je dirais même que cette audition de la première parole - dans la révélation inévitablement éthique du visage - résout l'autonomie où s'embarrasse pour une raison le concept même de la révélation.

L'altruisme est en effet le seul enseignement qui enseigne l'enseignement lui-même.

La question de savoir si je reconnais la divinité de Dieu ou de mon maître, à cause de l'enseignement qu'il me délivre (et si par conséquent l'enseignement est d'abord confronté avec la lumière naturelle - ce qui correspond à la position traditionnelle de la philosophie) ou si je reconnais l'excellence de mon enseignement à cause du Dieu ou du maître qui me l'enseigne (et qui s'est imposé à ma confiance d'une façon étrangère à toute lumière naturelle - ce qui correspondrait à la foi puisant dans la révélation).

Cette alternative est surmontée dans la position où Autrui ne se présente pas comme l'auteur d'un enseignement qu'il cautionne mais où sa présentation est ipso facto cet enseignement - où elle se produit dans mon obligation à son égard, où cette obligation n'est pas une violence ou une emprise, mais précisément une signification, une vérité. Et inversement, la première vérité, ou la première signification, ne serait pas possible si Autrui ne se présentait à moi en maintenant toute la distance de son étrangeté. 
Une lumière naturelle qui serait purement intérieure - n'est pas possible comme pensée.

Je ne choisis pas l'enseignant à cause de l'enseignement vrai, ni l'enseignement à cause de l'enseignant. La vérité de l'enseignement est précisément dans l'obligation éthique à l'égard de l'enseignant - et l'épiphanie de l'enseignant est déjà son enseignement.

Le décalogue commence par le Je de la théophanie. Un vieux apologue juif - cité il y a quelques années par le professeur Scholem dans la revue Eranos - veut que Dieu sur le Sinaï n'ait dit que ce premier Je et un autre apologue surenchérit: il n'aurait dit que la première lettre de ce Je - ce qui en hébreu est la lettre aleph - c'est-à-dire une lettre qui précisément ne se prononce pas. Mais tout l'enseignement du Décalogue est déjà dans cette Théophanie inaudible. C'est pourquoi un autre apologue prétend que jamais Dieu n'est descendu sur terre et que jamais Moïse n'était monté au ciel. Dieu avait simplement plié les cieux comme une couverture, les a étendus sur le Sinaï et s'est trouvé ainsi à la fois dans les cieux et sur la terre.

Il existe un point de convergence entre la philosophie et la religion, entre l'immanence et la transcendance. (sans cela le dieu des philosophes et le dieu d'Abraham d'Isaac et de Jacob ne convergera pas) C'est le minimum philosophique de la religion et le minimum religieux de la philosophie. Voilà où se situe la première parole de Dieu.

Qu'en est-il des autres paroles? Elles sont en dehors de la philosophie. Ou du moins étaient en dehors de la philosophie. Car je me demande si elles doivent rester dehors depuis que Heidegger a persuadé à tant de jeunes filles, à tant de jeunes hommes et même à tant de philosophes que l'être de l'étant s'est révélé dans les fragments des présocratiques ou dans les poèmes de Hölderlin, de Rilke, de Georg et de Trakl, que les philosophes doivent penser l'être à partir de ces textes.

Cela ne devrait-il pas nous encourager a chercher philosophiquement la parole de Dieu vivant dans les psaumes ou dans Isaïe fussent-ils comme nous l'enseigne la critique composés de fragments disparates et appartenant à des auteurs multiples - mais qui n'en parurent pas moins divin à travers une histoire européenne multimillénaire?

\section{Fécondité}

Je ne pensais nullement à une idée naturaliste en abordant la fécondité. Elle reste pour moi liée à la famille - Mais en réalité mon effort a consisté à présenter la fécondité comme une dimension de l'existence du Moi. En 
aucune façon il ne s'agit (pas) d'une vie de l'espèce qui se sert de l'existence personnelle pour mieux se manifester. Ce qui est la conception raciste de la fécondité. Que le moi lui-même puisse être extérieur à soi sans que ce soit un Autre, - voilà l'aventure de la trans-substantiation, plus étonnante qu'un passage d'un avatar à l'autre. Que l'autre qui est mon fils puisse être radicalement différent de moi, n'est pas contre ma position, mais conforme à elle. J'ai cité moi-même un verset d'Isaïe où la paternité s'affirme horrifiée devant la radicale étrangeté du fils. Ce n'est pas sur un contenu quelconque que serait fondée la filialité - elle est une reconnaissance de soi dans l'autre indépendante de tout contenu. Là encore je maintiens le terme formel. Rien n'empêche d'ailleurs par le retour de l'enfant prodigue. Mais la possibilité même de l'enfant prodigue confirme l'indépendance de la filialité et de la paternité à l'égard du contenu.

\section{Le mal}

Le problème du mal - vous l'avez dit - c'est le problème du meurtre et pourquoi sommes-nous tentés de commettre le meurtre? A cause de l'impérialisme du Moi. Parce qu'il existe un être séparé nécessaire à l'infinition de l'infini et à la production même du Bien. Dans un système où l'être équivaut à la totalité - on ne peut comprendre que par une chute, elle-même incompréhensible à partir d'une totalité - qu'il puisse y avoir seulement un être séparé. Et dans ce sens mon entreprise répond implicitement au problème du Mal.

Quant aux formes ténébreuses qui se profilent dans l'intersubjectivité elle-même, elles sont le fruit du totalitarisme politique - contre lequel la critique de l'idée de la totalité - c'est-à-dire toute ma thèse - est une protestation. Quant au tentateur - je ne crois pas que cette notion puisse se soutenir sans contradiction. Pouchkine - Lermontov.

\section{Une civilisation exceptionnelle}

Tout mon travail consiste à distinguer la signification du visage de la signification culturelle. L'homme - partout directement accessible - n'est certes pas un être abstrait du concret culturel, le genre homme dont tous les individus particuliers seraient les individus. Ce n'est pas l'abstraction du genre que l'on saisit dans chaque homme, mais le concret du visage à travers 
lequel l'homme se présente non pas comme une humanité en général, mais précisément comme cet homme-ci. L'humanité est un concept qui n'a pas de compréhension, mais l'extension seulement. Ce qui constitue la droiture de sa présence, c'est précisément l'obligation morale - l'impératif qui me vient de lui: «tu ne commettras pas de meurtre», indépendant des cultures.

Dira-t-on cependant que le «tu ne commettras pas de meurtre» n'est pas un ordre séparable des cultures. Le meurtre se définit par rapport à une loi. Et une loi ne peut-être - à moins de se ramener à un principe universel au sens kantien - que l'expression d'une culture.

A quoi je réponds: c'est «le tu ne tueras pas» et non pas le «tu ne commettras pas de meurtre» qui s'inscrit sur le visage. La civilisation occidentale - gréco-judéochrétienne - qui est précisément la civilisation qui annule les civilisations historiques - pousse la loi jusqu'à l'annulation de la loi puisque derrière la justice elle conçoit le pardon, puisqu'elle recule devant la justice tout court - devant la justice des raisons pures. Possibilité et tentation du pardon. Déchirement entre justice et pardon.

\section{Natures simples}

Pourquoi commencer par la transcendance, par le visage? Pourquoi ne pas partir du sujet qui doit constituer un objet? Pourquoi ne pas partir de la représentation de l'objet qui portera en elle (ou qui ne saura pas porter) des indices d'une existence extérieure et indépendante ce qui attesterait autrui?

La méthode consiste à reconnaître que ces points de départ prétendus naturels - sont déjà par eux-mêmes conditionnés. Que le sujet enfermé en lui-même est le sujet tel qu'il s'aperçoit dans une maison, que la représentation de l'objet est une manière de se sentir dans l'être, également conditionnés. Ce sont des significations et non des atomes de l'évidence. La signifiance du visage, le désir d'Autrui, la trans-ascendance, la conscience morale qui consiste à mettre en cause sa liberté et de la soumettre à autrui - sont des significations aussi immédiatement saisissables que les prétendus points de départ naturalistes ou idéalistes. Leur privilège ? La possibilité de rejoindre et de situer les autres. Juger de ce privilège, c'est juger du succès de cette entreprise.

C'est le grand apport de la phénoménologie que d'avoir reconnu dans les notions profondément simples, dans les atomes d'évidence des horizons où ils se placent et qui doivent être ramenés à l'évidence. Mais en réalité c'est Hegel qui nous a appris à dater en quelque manière les évidences éternelles. Mais en fait c'est la philosophie critique qui date cette manière. 
La découverte du transcendantal a été la découverte du caractère conditionné de l'objet. Chez Descartes existent encore des natures simples - des atomes d'évidence.

\section{Vérité et événement}

Tantôt j'affirme l'essence de la transcendance dans la vérité et tantôt je conteste ce lien entre vérité et métaphysique. Je l'affirme en tant que vérité suppose un être séparé dont le lien avec l'être n'est pas un lien d'intégration, en tant que l'être humain est séparé. La séparation à l'égard de l'infini est autre chose même que la présence dans un milieu. La présence dans un milieu est le côté positif de sa séparation, puisque le rapport avec ce milieu est jouissance. Je l'affirme en tant que vérité signifie relation avec l'extériorité. Je l'affirme en tant que vérité maintient l'idée de liberté dans la violence de l'opinion et de la guerre. Je l'affirme en tant que vérité comporte critique - c'est-à-dire méfiance - à l'égard de soi, c'est-à-dire la saisie de soi en deçà de son être - mais où saisie n'est pas possession (représentation) mais la mise en question de sa liberté ou éthique.

Je conteste que l'essence de la transcendance est la vérité car par vérité on entend la mise en lumière. Or ce n'est pas l'événement originel de la révélation laquelle est commandement éthique du visage (qui ne suppose pas vision). La production du visage n'est pas sa mise en lumière, mais son ordre. (C'est par analogie que je l'appelle lumière où l'on voit la lumière). Dans la mesure où les événements de l'être en dehors de la causalité ne sont pas dévoilement mais peuvent être nocturnes, comme par exemple la relation de la fécondité qui est au-delà du possible. (Chez Kant le fondement obscur de l'entendement et de la sensibilité - toute l'action transcendantale de constitution de l'objet n'est pas une œuvre de dévoilement).

Mais il reste tout de même le problème de l'universalité de la philosophie qui peut éclaircir même l'obscur événement de l'être. La clarté n'est pas à la mesure de l'événement - la philosophie n'est pas au-delà de la vie. L'explicitation pour se préserver contre la pensée totalitaire (critique de la raison). Car la métaphysique n'est possible que par la séparation laquelle s'accomplit par la représentation en laquelle peut (virer?) toute la vie. L'explicitation est l'accomplissement de la transcendance en tant qu'elle confirme la séparation indispensable à la transcendance. La philosophie est à la fois l'extrême possibilité humaine et l'avant-dernière. 


\title{
Alternance entre représentation et éthique
}

Mais la suprématie de l'Autre se manifeste peut-être dans le fait que la demeure elle-même, et l'identité du chez soi - n'est possible qu'à cause d'un accueil que l'Autre sous les espèces du féminin - réserve à l'être séparé. De sorte que la séparation elle-même se doit à l'Autre - quoi que cette dépendance n'ait rien de dialectique.

Si l'essence de la philosophie consiste à remonter en deçà des certitudes obtenues dans la spontanéité - si elle est ontique - l'épiphanie du visage serait le commencement même de la philosophie. Thème d'hétéronomie qui rompt avec une tradition très vénérable.

Qu'est-ce que la philosophie après tout?

Nous pensons concrètement - à travers la culture - la philosophie s'en rend compte et ne peut pas annuler cette vie à travers la culture, mais elle consiste aussi à découvrir que cette culture est un langage. Elle ne le prend pas complètement au sérieux et se rend compte de la convergence de langages divers - vers l'absolu. Elle consiste à dire dans un langage spécial un absolu qui n'est pas une abstraction par nous provoquée, mais qui commande toutes les cultures - qui s'absout soi-même de l'être. C'est le visage de l'Autre - dominant les cultures - Elle affirme l'Ethique. - Elle préserve de l'idolâtrie - Elle est monothéisme - Elle est en fin de compte religieuse.

\section{Le triomphe de la justice dans la fécondité = Eternité du peuple juif.}

\author{
$* * *$ \\ Texte en russe de Crime et châtiment, édition Ladynikof, \\ pages 701-702.
}

«Il regardait ses compagnons des travaux forcés et s'étonnait comme ils aimaient tous la vie, comme ils lui attachaient du prix. Précisément, il 
lui semble qu'à la maison de correction qu'on l'aime encore plus, qu'on l'apprécie encore plus qu'en liberté. Que de souffrances et de tortures terribles certains d'entre eux n'avaient supportées, comme par exemple les vagabonds! Est-ce que peut avoir pour eux tant d'importance un je ne sais quel rayon de soleil, la dense forêt, quelque part dans un trou inconnu, une source froide remarquée il y a trois ans, et que le vagabond rêve de revoir, comme une maîtresse qu'il voit en rêve, avec l'herbe verte autour et un oiseau qui chante dans le buisson", Crime et châtiment, épilogue, édition Ladynikof, p. 701.

"Où ai-je donc lu qu'un condamné à mort dit et pense à une heure de la mort que s'il devait vivre quelque part sur une hauteur et sur un palier si étroit qu'il puisse tout juste placer les deux pieds - et que tout autour béent des abîmes, s'étendent l'océan, les ténèbres éternelles, une solitude éternelle et une tempête éternelle - et s'il devait rester ainsi debout sur un mètre d'espace toute une vie, mille ans, une éternité - il vaut mieux vivre ainsi que de mourir tout de suite. Rien que vivre, vivre, vivre. Quelle que soit la vie pourvu qu'on vive! Quelle vérité. Mon Dieu quelle vérité! L'homme est ignoble! Mais est ignoble celui qui le traite d'ignoble pour cela - ajoutait-il une minute après. » Crime et châtiment, édition Ladynikof en russe, Berlin, 1922, p. 206.

Le plan d'Autrui surgit ensuite - Mais cette condition paradisiaque existe.

\section{Les divers points fondamentaux}

1) Distinction entre Désir et besoin.

2) Au-delà de l'être - pas néant mais le surplus du social. L'excellence de la séparation.

3) Le visage en tant que signification première: la signification, c'est l'éthique - à partir duquel le monde prend un sens comme ce qui peut être offert. Le paysage premier: désert.

4) L'être séparé - le pour soi de la jouissance; le pour soi = apologie.

5) La mort n'est pas une solitude (déjà Socrate n'est pas seul à mourir).

6) L'isolement $=$ jouissance.

7) Le Moi c'est le Même, comme chez Fichte.

8) Derrière l'ontologie - dépasser l'être dans la relation éthique avec l'étant.

9) Liberté: rester le Même et ne pas avoir à se soumettre à l'Autre. Dans ce sens - et dans la mesure où la Raison assimile tout, liberté = raison. 
Pour moi : cette liberté qui assimile tout est impérialiste et injustifiée. Elle se découvre telle en présence de l'Autre. Elle se découvre aussi comme un enchaînement à soi et monotone: destin. L'autre peut lui donner l'investiture (il y a une aliénation qui la libère de soi). L'autre lui découvre la distance et le temps, de sorte que rien n'est plus définitif en Moi. Plus de destin. Fécondité $=$ liberté.

10) Corrélation des notions comme enseignement, maîtrise, jugement, bonté, désir, éthique, commandement.

11) Le point de vue dit «subjectif» est dépassé non pas dans sa suppression - mais dans sa confirmation au sein de sa collaboration au jugement même qui se porte sur lui : lorsque à sa peur de sa mort se substitue la peur pour le meurtre. Le subjectif est nécessaire à l'aliénation qu'est en fin de compte le jugement lui-même. Aliénation hostile et aliénation pacifique.

12) La signification, c'est l'inadéquat = ce qui parle, ce qui n'est pas simplement compris, mais appris. Signifier - c'est enseigner. Celui qui signifie - (?) ou signifie un ordre.

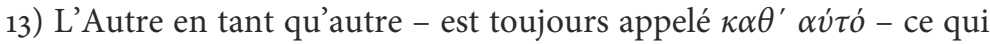
peut faire croire qu'il est encore le Même. En réalité ce n'est pas la même identité que l'identité du Moi - ou si l'on veut c'est le fait que quelque chose d'identique est hors de moi - selon soi-même, mais autre que moi-même: c'est là le bouleversant de la rencontre. Le Moi inséparable de cette altérité souscrit toute altérité. Être autre = être étant = être quelqu'un = être homme. C'est cette altérité du moi qui en constitue la force d'étant.

14) La réflexion suppose une mise en question de soi, une attitude critique - qui se produit sous l'autorité de l'Autre.

15) Conscience de l'indignité - scrupule qui ronge la conscience. (Ronger une conscience?) est un mode opposé à la prise de conscience.

16) Aborder Autrui par l'entremise de chose = non pas par leur médiation mais en les donnant. Les choses condition des mains pleines. «Vous ne vous présenterez pas devant ma face les mains vides».

17) Généralité et communauté d'appartenance. Le général - ce qui ne peut être approprié - dépouillé de tout attribut s'offrant à la possession.

18) Philosopher c'est remonter en deçà de la liberté, découvrir l'investiture qui libère la liberté de l'arbitraire.

19) La nature de soi qui s'exprime dans une volonté s'exprime dans une volonté arbitraire comme cette nature (même s'il se distingue du caprice).

2o) Je définis comme Fichte le Même par le Moi - et par là j'exclus la totalité.

21) La notion de vivre de... ou jouissance.

22) La représentation affirme le caractère radical de la séparation: la souveraineté du Même capable d'absorber dans une totalité tout Autre 
- et dont la jouissance est l'ultime noyau. Certes, cette souveraineté est conditionnée par la Demeure et l'Autre féminin qui y attend (il existe des êtres qui ne peuvent pas contempler: les pauvres). La générosité de la représentation repose sur la possession et la Demeure - par le visage d'autrui qui rend possible l'édification de la maison; mais (s'il ne conditionne pas le Même?) anti-thétiquement.

Nous ne présentons pas une philosophie de la réflexion - une analyse de la conscience dans une situation donnée qui érige en absolu, c'est-à-dire en structure permanente (et indépassable par l'histoire) les données d'une subjectivité particulière. Tout au contraire nous dénonçons la subjectivité dans son incontrôlable arbitraire - qui est pour nous le Même avant qu'il ait reçu la révélation de l'Autre. Cette contingence de la subjectivité n'est pas d'ailleurs pour nous d'une insuffisance purement logique. Elle est positivement la possibilité du crime en tant qu'expansion sans entraves dans un monde des subjectivités à données particulières différentes et que l'on appelle colonialisme. Cela aura été le grand mérite de la phénoménologie que d'avoir précisément montré le caractère conditionné des structures que l'idéalisme prenait pour des natures simples et pour des points de départ d'une philosophie éternelle.

Nous présentons la subjectivité comme s'érigeant seulement dans la relation avec Autrui dont la manifestation est première et qui n'est pas essence - contrairement à l'objet - est inadéquate à la subjectivité et qui reçoit de lui des commandements. Dans ce sens l'aliénation du Même n'est pas un événement regrettable et un danger que court la subjectivité, mais la grande affaire de la subjectivité. (Ce sont précisément les philosophes qui insistent sur l'importance de l'histoire dans la détermination du Même qui conservent la nostalgie de la subjectivité abstraite, non altérée par l'Autre - mais pour qui le lacis des relations historiques est à la fois un obstacle de cette liberté du Même et les voies qui y mènent, qu'il faut considérer pour que la liberté du Même puisse se produire concrètement et non pas abstraitement telle qu'elle se joue d'emblée dans le Même). Mais nous soutenons que cette aliénation peut se produire de deux manières - soit par la violence - guerre et corruption - et qui en réalité n'aliène la subjectivité qu'au regard objectif qui l'aperçoit dans une totalité - que ce regard soit celui d'autrui ou celui de moi-même. Le moi aliéné de cette façon est esclave - qui est une condition objective, mais qui n'est jamais une condition du pour soi, toujours au bord de la révolte et de l'affranchissement et de l'évasion - toujours à l'état de frustration, condition inhumaine. 
L'autre forme de l'aliénation - est précisément l'altruisme. Et qui exige précisément la participation du Même et qui l'investit en tant qu'être séparé et particulier et irremplaçable et disposant des ressources infinies que l'altruisme exige. Et qui est la façon dont l'aliénation au premier sens $d u$ terme peut-être surmontée elle-même.

Ce qui donne le droit de faire l'analyse de la subjectivité en face de l'autre avant les manifestations historiques, c'est l'affirmation

a) que sous la forme historique il n'existe pas d'aliénation sinon dans l'objectivité, qu'il n'y a pas d'esclavage subjectivement. Que l'esclavage est toujours en soi, mais jamais en soi et pour soi.

b) Que l'analyse que nous poursuivons sous forme de réflexion n'est pas l'analyse d'une conscience qui s'interroge sur sa liberté constituante, mais d'une conscience qui se met en question - qui est une conscience dite morale.

c) Que l'aliénation altruiste consiste à rencontrer par delà l'histoire et ses modifications le visage qui tranche sur ces modifications et qui dans ce sens est un absolu. Que la relation avec Autrui dans son unicité se refusant à tout genre comporte l'universalité de l'humanité qui n'est ni l'universalité du genre, ni l'universalité formelle (car tous les hommes différents constituant une totalité réfractaire à la totalisation sont concernés lorsque nous abordons le visage d'un homme avec lequel les autres sont en relation d'individu à individu antérieurement à la totalité). Il y a des relations qui précèdent la culture.

Ad a) Nous nions l'aliénation dans l'histoire et dans la collectivité qui serait autre chose qu'un fait de l'objectivité. Que les événements de l'histoire puissent modifier la subjectivité en tant que matière sur laquelle cette subjectivité agit - c'est incontestable. Que les événements de l'histoire puissent y jouer un rôle transcendantal - cela a été montré par le développement du hégélianisme et par la phénoménologie. Certaines idées ne seraient pas possibles sans qu'une certaine situation historique se produise. Non pas comme produit de cette société, mais comme constituées transcendentalement par cette situation. Il fallait par exemple l'apparition d'une société industrielle pour que l'humanité puisse être explicitement pensée sur un plan planétaire, etc.

Mais ces deux points ne peuvent pas mettre en doute la valeur de la méthode phénoménologique dite de réflexion et menée antérieurement à l'histoire.

Tout change avec l'aliénation pensée d'une façon ontologique. Mon livre est précisément l'affirmation que l'aliénation n'est pas ontologique - que la présence d'Autrui à moi est une garantie du Même en tant que Même. 
Que la guerre et le commerce annoncent une philosophie de l'aliénation et de la totalité, mais que la conscience de l'aliénation n'est pas l'aliénation ontologiquement - car la conscience de l'aliénation en tant que conscience est déjà antérieure à l'aliénation, mais que cette conscience de l'aliénation se refuse à l'aliénation précisément parce qu'elle peut être altruiste et que en Autrui elle trouve quelque chose d'autre que la conscience qui arrête la thèse «nous sommes ce dont nous avons conscience». Nous sommes la mise en question de la conscience qui n'est pas un événement de la conscience, mais «l'idée de l'Infini».

Chez Husserl il n'y a pas d'aliénation - et c'est pourquoi il admet une philosophie transcendantale. Tout se constitue en fin de compte pour un moi extérieur à l'histoire, c'est-à-dire pour un moi absolument non-aliéné. La réduction n'est possible que dans l'hypothèse de la non aliénation. Notre façon de concevoir cet arrachement à l'histoire et à la culture est différente. Nous avons cherché à la conscience toujours déjà engagée dans l'histoire et aliénable - la relation avec Autrui qui n'est pas conscience, mais moralité.

\section{Création \\ Le Bien au-delà de l'Être}

Dans la création il n'est pas question du moi de la production ou de la causalité absolue que ce concept contient théologiquement. Il s'agit avant tout de fixer par un terme

a) la structure de l'être où la totalité ne reconstitue pas, où quelque chose peut exister en dehors de la totalité.

b) La possibilité pour un être de se mettre en question et de chercher au-delà de son origine, de ne pas partir de son origine comme d'un absolu (de rendre intelligible la naissance) (qui cesse d'être la violence par excellence), c'est-à-dire la possibilité de la moralité.

c) ex nihilo - en tant que refus d'une matrice commune des choses qui reconstitue la totalité du pour soi, sous un être causa sui.

Il faut souligner la signification philosophique des concepts religieux. Il faut partir de la signifiance propre de ces concepts. (?) Contre le refus du théologique. Dans son énoncé mythologique. Dieu existe-t-il ? Etc. Miracle, irrationnel. 
La notion de visage répond à une double préoccupation:

1) poser la personne avant de poser l'universalité (Renouvier contre Kant).

2) Répondre à l'équivoque raison et être raisonnable. Le visage c'est la découverte de l'être raisonnable et non pas d'une raison. («Discours vivant et animé», Platon, Phèdre 275a). Découverte de l'homme. Réponse aussi à la question de la multiplicité des êtres raisonnables = la raison est une - raison (?) société et cependant société suppose raison. Même réponse à la question: que peut signifier être raisonnable? Raison - n'est pas un attribut: l'expression être doué de raison m'a toujours paru terriblement équivoque. L'être raisonnable: un visage. 


\section{Annexe 1 Résumés de thèse par Emmanuel Levinas}

\section{Totalité et Infini ${ }^{14}$}

Thèse principale soutenue en vue du Doctorat ès Lettres par Emmanuel Levinas, le 6 juin 1961. A paraître dans "Phaenomenologica», collection publiée sous le patronage des centres d'Archives-Husserl, chez Martinus Nijhoff, à La Haye (Pays-Bas).

En un sens, philosopher revient à déterminer tout Autre à partir du Même. La recherche de la vérité est la manière dont cette détermination s'accomplit. Elle consiste à retailler l'Autre à la mesure du Même, à retrouver en soi la mesure de l'Autre. La philosophie se définirait par l'adéquation, l'autonomie, l'a priori, qui suppriment la multiplicité en l'intégrant dans une totalité où vient prendre place le philosophe lui-même. Mais l'adaptation de l'Autre à la mesure du Même dans la Totalité, ne s'obtient pas sans violence - guerre ou Administration - lesquelles aliènent le Même lui-même. Le Moi ne reconnaît pas alors sa volonté dans les conséquences de son vote.

Mais la philosophie comme amour de la vérité aspire à l'Autre comme tel, à l'être distinct de son reflet en Moi - elle recherche sa Loi, elle est l'hétéronomie elle-même, elle est méta-physique. Chez Descartes, le Moi qui pense, possède l'idée de l'infini: l'altérité de l'Infini ne s'amortit pas dans l'idée, comme l'altérité des choses finies dont, d'après Descartes, je peux rendre compte par moi-même. L'idée de l'infini consiste à penser plus qu'on ne pense.

Cette description négative prend un sens positif, qui n'est plus dans la lettre du cartésianisme: une pensée qui pense plus qu'elle ne pense, qu'est-ce sinon le Désir? Désir qui se distingue de l'indigence du besoin. Le Désiré ne le comble pas, mais le creuse.

La phénoménologie du rapport avec Autrui, suggère cette structure du Désir ; analysé comme idée de l'Infini. Alors que l'objet s'intègre à l'identité du Même, Autrui se manifeste par la résistance absolue de ses yeux sans défense. Il vient d'une dimension de hauteur. L'inquiétude solipsiste de la conscience se voyant, dans toutes ses aventures, captive de soi, prend fin ici. Le privilège d'Autrui par rapport à Moi - ou la conscience morale - est la percée même vers l'extériorité, qui est aussi une percée vers la Hauteur.

14. Résumé de thèse envoyé à l'Université de Paris-Sorbonne après la soutenance de thèse pour publication dans les Annales de l'Université. 
L'épiphanie de ce qui peut se présenter aussi directement, aussi extérieurement et aussi éminemment - est visage. L'exprimant y assiste à l'expression, "porte secours à lui-même», signifie, parle. L'épiphanie du visage est langage. Autrui est l'intelligible premier. Mais l'infini dans le visage n'apparaît pas comme une représentation. Il met en question ma glorieuse spontanéité de force qui va. Ma liberté se découvre meurtrière et usurpatrice. Mais cette découverte n'est pas un dérivé du savoir de soi. Elle est de part en part hétéronomie. Devant le visage j'exige toujours plus de moi-même; plus j'y réponds et plus les exigences augmentent. Ce mouvement est plus fondamental que la liberté de la représentation de soi. La conscience éthique n'est pas, en effet, une variété particulièrement recommandable de la conscience, mais la contraction, le retrait en soi, le systole de la conscience tout court.

L'orientation vers la hauteur de l'Autre - ainsi décrite est comme un dénivellement dans l'être lui-même. L'au-dessus n'indique pas une néantisation, mais un "plus qu'être», meilleur que le bonheur, de la relation sociale. Sa "production" serait impossible sans la séparation, laquelle ne saurait se réduire à un pendant dialectique de la Relation avec Autrui. Car la dialectique de la séparation et de l'union se joue déjà dans une totalité. Le principe de la séparation, est fourni non pas par le malheur de la solitude, déjà tournée vers autrui, mais par le bonheur de la jouissance. Dès lors il devient possible de soutenir un pluralisme qui ne se réduit pas à une totalité.

Dire qu'Autrui, se révélant par le visage, est le premier intelligible, avant les cultures, leurs alluvions et leurs allusions, c'est aussi affirmer l'indépendance de l'éthique par rapport à l'histoire. Montrer que la première signification surgit dans la moralité - dans l'épiphanie quasi-abstraite du visage dénué et dénudé de toute qualité - absolu - s'absolvant des cultures, c'est tracer une limite à la compréhension du réel par l'histoire et retrouver le platonisme.

\section{Thèse de doctorat es Lettres (Doctorat d'Etat) ${ }^{15}$ Soutenue le 6 juin 1961}

Thèse principale: «Totalité et Infini», par Emmanuel Levinas

A paraître dans «Phaenomenologica», collection publiée sous le patronage des centres d'Archives-Husserl, chez Martinus Nijhoff, à La Haye (Pays-Bas).

15. Résumé de thèse demandé par l'université pour le French Bibliographical Digest. 
Hegel affirme la corrélation entre les idées de vérité, de totalité et de liberté. Tout Autre heurte le Même ou le Moi. Dans la totalité seulement apparaît la vérité des termes isolés, et la liberté de l'autonomie régit alors le Tout.

La philosophie serait-elle née de l'Allergie? Ou, la vérité se réfère-t-elle aussi à l'être, à ce qui est autre que soi-même, autre que le Même, à l'expérience de l'absolument Autre, à sa loi ? Elle est alors hétéronomie et méta-physique. Le Bien platonicien, au-delà de l'être, l'idée de l'infini de Descartes, débordent la Totalité. L'idée de l'infini, qui pense plus qu'elle ne pense, peut s'interpréter comme Désir distinct du Besoin. Rien ne saurait combler ce Désir; le Désiré le creuse au lieu de le satisfaire. Situation qui est concrète dans le rapport avec Autrui. Le Désiré n'est pas objet. Un objet s'intègre à l'identité du Même. Autrui, le Désiré, résiste à l'impérialisme du Même par la nudité totale de ses yeux sans défense. Son logis est «Tu ne tueras point». Il ouvre une dimension de Hauteur. L'épiphanie de ce qui peut se présenter aussi directement et aussi éminemment, est fixé par le terme: visage. En lui, l'exprimant assiste à l'expression, "porte secours à lui-même ", parle, signifie. Le visage est l'intelligible premier. Le premier intelligible est éthique. Mais cette signification première n'est pas une représentation. La révélation de l'Autre, consiste à me mettre en question. Se mettre en question, n'est pas une variante du «se connaittre». Elle consiste non pas à être pour soi, mais à accroître sans cesse, en face d'Autrui, les exigences à l'égard de soi, mouvement plus fondamental que la liberté.

L'idée de l'infini, l'orientation vers la hauteur à travers le visage - est comme un dénivellement dans l'être lui-même. On peut dans une telle structure ontologique apercevoir un pluralisme, irréductible à une Totalité. Soutenir que la première signification surgit dans l'éthique - dans l'épiphanie quasi-abstraite du visage dénué et dénudé, s'absolvent des cultures - absolu - c'est placer l'intelligible avant la Culture et indépendamment de l'Histoire et, par conséquent, retrouver l'essentiel du platonisme. 


\section{Annexe 2 Rapport de Gabriel Marcel ${ }^{16}$}

Une période plus longue de maturation eut été nécessaire...

Les premières questions que je voudrais vous poser portent bien entendu sur certaines conceptions fondamentales. Je regrette que vous n'ayez pas commencé par présenter une critique des conditions dans lesquelles il est légitime de penser la totalité - une critique qui pourrait être comparée à la critique de l'idée de néant chez Bergson. Vous vous référez à l'ouvrage de Bergson, mais c'est là une indication insuffisante. Il se peut du reste que nous ayons à revenir sur ce point.

C'est sur le rapport de l'être, du tout et de l'infini que je voudrais vous demander des explications. Je me référerais ici à trois passages qui me paraissent très importants. D'abord celui de la page 17 . Vous venez de critiquer l'ontologie heideggérienne. Mais vous vous exprimez de la manière que voici:

«Philosophie du pouvoir, dites-vous, l'ontologie comme philosophie première qui ne met pas en question le Même, est une philosophie de l'injustice ${ }^{17}$. Je note en passant que cette préoccupation de l'injustice et de la guerre, d'une part, de la bonté et de la paix de l'autre, est chez vous fondamentale, et qu'elle sous-tend littéralement votre livre. Mais je voudrais savoir si vraiment pour vous, l'ontologie en tant que telle est philosophie de l'injustice, en tant, je suppose, qu'elle affirme l'auto-suffisance de l'être. Certains passages paraissent aller dans ce sens, en particulier page 76 où vous évoquez Platon. La place du Bien, au-dessus de toutes essences, dites-vous, est l'enseignement le plus profond - l'enseignement définitif non pas de la théologie, mais de la philosophie. Le paradoxe d'un infini admettant un autre être en dehors de soi qu'il n'englobe pas et accomplissant grâce à ce voisinage d'un être séparé son infinitude même - en un mot le paradoxe de la création - perd dès lors de son audace. Ceci peut paraître relativement clair. Je voudrais néanmoins être sûr que l'être est ici en quelque façon identifié à la totalité et que justement l'ontologie est refusée en tant que philosophie de la totalité. Mais je pense que certains textes essentiels de votre livre ne s'accordent nullement avec cette interprétation. Ne dites-vous pas dans la préface que la «face de l'être qui se montre dans la guerre, se fixe dans le

16. Rapport manuscrit de 13 pages envoyé à Levinas par Gabriel Marcel lui-même, en vue de la soutenance.

17. Toujours la pagination de l'édition de Totalité et Infini de 1961 chez Martinus Nijhoff. 
concept de totalité qui domine la philosophie occidentale» (page X) ? La face de l'être: il y aurait donc une autre face! Si l'on s'engageait dans cette direction on serait conduit, semble-t-il, à admettre qu'il y a un versant de l'être qui est accessible à la pensée totalisante, c'est-à-dire à celle par laquelle l'altérité se résorbe en fin de compte dans le Même. Mais cette interprétation ne me paraît pas compatible avec le texte de la page 76 . Et surtout avec un texte extrêmement important qui figure dans la conclusion sous le titre «L'être est extériorité». Vous complétez de la façon suivante: «L'exercice même de son être consiste en l'extériorité et aucune pensée ne saurait mieux obéir à l'être qu'en se laissant dominer par cette expérience ${ }^{18}$. Comment cela peut-il s'accorder avec ce que vous dites page 251, l'être est, en tant qu'il est pour nous, une monade ${ }^{19}$. Je dois vous avouer qu'ici l'expression est loin de me satisfaire. L'exercice même de son être, dites-vous, c'est-à-dire l'être de l'être, mais ceci est dépourvu de toute rigueur: faudrait-il dire, ce qui serait plus acceptable, mais qui ne correspond peut-être pas du tout à votre pensée, de l'être de l'étant? D'autre part, je ne puis accepter qu'on dise l'exercice de l'être (sous-entendu de l'étant) consiste en l'extériorité. Vous prêtez ici à ce terme d'extériorité une signification qu'il ne peut pas avoir. L'exercice en question pourrait consister en une extériorisation, mais j'ai l'impression que cette phrase qui aurait un sens, dénaturerait votre pensée. Je n'accepte pas davantage qu'on dise d'une part que cet exercice obéirait à l'être et moins encore qu'il se laisse dominer par cette extériorité - ce dernier membre de phrase suffisait d'ailleurs à montrer que le terme d'extériorisation ne correspondait nullement à ce que vous voulez dire. En réalité, les lignes qui suivent rejoignent le thème central de votre ouvrage, c'est-à-dire le face à face qui s'établit dites-vous à partir d'un point, séparé de l'extériorité, ajoutez-vous, si radicalement qu'il se tient de lui-même.

Je suis persuadé à la vérité que l'emploi du mot extériorité est ici fâcheux, car il a avant tout pour nous une valeur de négation. Il s'agit de refuser - et vous le faites avec une ténacité qui force l'admiration toute philosophie de type par exemple spinoziste ou hégélien ou même ajouterais-je brunschvicgien qui tend à identifier être et intériorité, c'està-dire en fin de compte toujours à résorber l'autre dans le Même. Mais pouvez-vous empêcher que le terme d'extériorité soit interprété dans un sens spatialisant, alors que manifestement la relation, si c'est bien une relation - ce que l'on pourrait peut-être contester - sur laquelle vous ne cessez de mettre l'accent, doit être interprétée de façon non-spatiale.

18. Page 266. En fait Levinas n'écrit pas « expérience », mais « extériorité ».

19. Le texte exact de la page 251 est : «L'être en tant qu'être est pour nous monade ». 
Il y a là d'ailleurs un paradoxe très singulier. Je serais tenté de dire que vous vous efforcez continuellement et de façon presque acrobatique à dé-spatialiser ce que l'on pourrait appeler la distance et même l'intervalle. J'anticipe ici sur une remarque qui devrait être peut-être formulée plus tard, mais qui intervient nécessairement dans ce contexte. Vous tentez d'introduire une multiplicité non totalisable, une multiplicité qui ne peut pas être pour ainsi dire saisie synoptiquement. Toute votre pensée est anti-synoptique (ou anti-panoramique) et c'est d'ailleurs ce qui me paraît passionnant dans votre tentative. Mais il me paraît tout à fait évident, conformément à ce que j'indiquais il y a un instant, que cette multiplicité non totalisable doit être pensée, par là même, comme non spatialisée, je n'ose dire, bien entendu, comme extra-spatiale. Il y a là une difficulté que la plupart des esprits jugeraient sans doute insurmontable. Je n'irai pas aussi loin, mais en revanche je dirai que vous ne pouvez pas vous engager dans cette aventure, car c'en est une, sans prendre des précautions terminologiques auxquelles je n'ai pas l'impression que vous soyez suffisamment astreint. Dans le même sens, je serais d'ailleurs tenté de me demander si vous ne prêtez pas sérieusement le flanc à la critique par l'usage que vous faites des mots autrui et altérité. Tant que vous vous en tenez à l'opposition du vécu de l'autre, je crois que vous êtes hors d'état d'échapper à la dialectique que vous entendez précisément dépasser ou transcender. Je me demande si vous n'auriez pas été mieux inspiré en évitant de recourir aux termes autre et altérité, lorsque vous abordez votre domaine de prédilection, c'est-à-dire la philosophie du visage, du langage, ou du face à face. Pourquoi ne parlez-vous pas plutôt du prochain? Sauf erreur de ma part, il me semble que ce terme n'apparaît nulle part et pourtant il est appelé par le contexte. Je pense à vrai dire qu'il n'y a pas là une simple omission et que vous avez eu un motif précis pour ne pas employer ce mot, mais lequel!

Le terme "autrui» dont vous vous servez souvent soulève des objections: autrui, il me semble que c'est un peu le on dans l'autre, or, c'est justement sur le singulier en tant que tel que vous ne cessez de mettre l'accent. Ceci à vrai dire n'est pas très important.

En revanche je voudrais m'arrêter sur la façon dont intervient l'infini dans votre texte. Les premières indications que vous en donnez dans la préface ne me paraissent pas très éclairantes (page XIII) : «Dans l'idée de l'infini, écrivez-vous, se pense ce qui est toujours extérieur à la pensée " et un peu plus bas: «Le rapport avec l'infini ne peut certes pas se dire en termes d'expérience, car l'infini déborde la pensée qui le pense». Déjà dans ce texte vous usez de façon peut-être imprudente du mot extérieur : bien sûr vous vous référez à Descartes, mais je me demande jusqu'à quel point vous 
avez le droit de le faire. Si je ne me trompe, l'infini chez Descartes n'est pas séparable d'une philosophie du parfait qui me semble aussi éloignée que possible de la vôtre. C'est d'ailleurs là un point qui peut-être peut donner lieu à la discussion. Beaucoup plus loin, page 170, vous dites que l'idée de l'infini dépasse vos pouvoirs en les mettant en question. Vous ajoutez qu'elle ne vient pas de notre fond a priori et que par là elle est expérience par excellence. Ce qui, entre parenthèses, me semble en contradiction avec ce que vous avez dit dans la préface. Il me semble que cette affirmation n'a de sens qu'à condition de vouloir dire que l'idée de l'infini est non pas le contenu, mais si l'on peut dire le moteur de l'expérience, ceci rejoindrait alors un texte obscur, mais très important. Dans ce texte, page 281, vous écrivez ceci: «Nous avons décrit le désir comme la mesure de l'infini qu'aucun terme, aucune satisfaction n'arrête. Désir opposé à besoin ${ }^{20}$. Ici encore je dois vous dire que je vous chercherai querelle sur la terminologie. Il me semble que vous prenez désir dans le sens de l'eros platonicien. Je comprends à vrai dire très bien que vous n'ayez pas voulu traduire eros par amour, ce qui aurait donné lieu à de graves confusions. Mais le mot désir me paraît impropre. Nous pouvons ici difficilement faire abstraction de la tradition philosophique pour laquelle le désir est ordonné à la possession, même s'il va au-delà de toute possession déterminée. La phrase qui suit, page 281 , met en lumière un des paradoxes de votre pensée puisque vous dites que poser la métaphysique comme désir, c'est interpréter la production de l'être - désir engendrant le désir comme bonté et comme au-delà du bonheur. C'est interpréter la production de l'être, comme être pour autrui. Dans ces différentes formules, tous les mots pourraient donner lieu à critique. Je ne m'y attarderai pas, mais je dirai seulement qu'il est tout à fait singulier de vous voir dissocier aussi radicalement que vous le faites la jouissance qui figure dans l'ordre du Même et à laquelle vous consacrez d'ailleurs de très belles pages - et le désir. Même distingué du besoin, je me demande si le désir en tant que tel ne reste pas d'une certaine façon égocentrique, si la bonté ne procède pas toujours de l'agapè, celle-ci étant d'un ordre irréductiblement différent du désir.

Il y a là une question très importante et que nous aurons peut-être à retrouver plus tard. Mais pour revenir au point de départ, je me demande jusqu'à quel point il est légitime d'interpréter l'infini dans la perspective du désir. Vous parlez quelque part du mauvais infini comme si vous admettiez la distinction hégélienne. Il sera d'ailleurs bon que vous vous expliquiez sur

20. Le texte de Levinas est très légèrement différent. Nous conservons la citation telle qu'elle est reprise par Gabriel Marcel. 
ce point. Mais dans cette perspective-là, je veux dire celle de Hegel, il me paraît tout à fait évident, que le désir de quelque manière qu'on le définisse, est du côté du mauvais infini ou en tout cas en deçà du bon.

Je vois à vrai dire assez clairement qu'il s'agit avant tout pour vous, faut-il dire de désontologiser l'infini, de le penser comme transcendant à l'être. Seulement ici nous retrouvons l'équivoque que je signalais tout à l'heure. Ce serait beaucoup plus clair si pour vous l'être était la totalité. Mais au fond je pense qu'il n'en est nullement ainsi et qu'il y a peut-être même là une grave équivoque, puisqu'au fond vous défendez, semble-t-il, une conception extatique de l'être comme extériorité.

Cependant il est temps de passer à l'essentiel c'est-à-dire à votre philosophie du visage ou du face à face. Tout d'abord, dans la ligne de ce qu'on pourrait appeler votre pensée fondamentale, il faut, me semble-t-il, attacher une grande importance à des phrases telles que celles-ci : page 239: «L'extériorité définit l'étant comme étant. (...) Dans le visage se présente l'étant par excellence ${ }^{21}(. .$.$) et la signification du visage tient à la coïncidence$ essentielle de l'étant et du signifiant. La signification ne s'ajoute pas à l'étant. Signifier n'équivaut pas à se présenter comme signe, mais à s'exprimer, c'est-à-dire à se présenter en personne».

Je tirerai d'abord de ces passages cette conclusion qui me paraît très importante, qu'au fond dans la mesure où vous retenez - et où vous avez peut-être tort de retenir - l'opposition heideggérienne de l'être et de l'étant, vous prenez résolument parti pour l'étant, et cela peut-être pour cette raison profonde que vous craignez qu'une philosophie de l'être ne glisse, comme c'est le cas à tout le moins dans les écrits récents de Heidegger, vers une philosophie du neutre - ce neutre que vous abhorrez et que je n'aime pas beaucoup plus que vous. Mais ici les différences commencent - il serait en effet inexact, je crois, de vous attribuer une thèse personnaliste, et à cet égard, il est important de discerner ce qui vous sépare de Kant.

Au premier abord, on pourrait être plutôt frappé des ressemblances, je vise ici surtout l'idée kantienne de la personne ou du sujet éthique comme fin en soi - et d'une façon générale l'idée du primat de l'éthique est vôtre, ne vous apparente-t-elle pas au kantisme? Mais il y a entre lui et votre pensée cette différence capitale que chez Kant les personnes apparaissent respectables en tant qu'elles participent à la raison ou si l'on veut à l'universel. Or l'idée de cette participation vous est contraire; vous mettez l'accent sur la singularité, sur la différence absolue. Se tromperait-on en vous disant que cette absolutisation de ce que j'appellerais assez volontiers l'intervalle ou

21. Cette proposition se trouve en fait après le reste du texte. 
si vous voulez l'altérité radicale, est comme une transposition de celle qui chez Kierkegaard porte sur la subjectivité en tant que telle?

Seulement, dans ces conditions, la difficulté majeure consiste à comprendre comment et pourquoi le face à face prend valeur éthique; ceci, vous cherchez à l'éclairer par des spécifications telles que l'étranger, la veuve, l'orphelin (l'hospitalité, la compassion) ou encore le face à face avec le maître, le rapport enseignant-enseigné. Il y a même un passage où vous juxtaposez ces deux significations sous une sorte d'accolade. J'aperçois cependant dans tout cela de grandes difficultés. Sont-ce là des différences spécifiques à l'intérieur d'un même genre? Il me paraît également difficile de l'admettre et de le nier. Et d'autre part, pouvez-vous prétendre que tout visage en tant que tel est signifiant. Je crains d'ailleurs que cette idée du signifiant comme expressif ou de la signification distinguée de la Sinngebung ne soit en elle-même assez confuse. Pouvez-vous nier qu'il y ait dans l'expérience tel visage qui ne me dit rien, qui non seulement est pour moi vide de signification, mais qui n'éveille en aucune manière ce désir dont nous avons déjà parlé et qui est irréductible à la convoitise. Mais irez-vous jusqu'à porter une appréciation éthique de l'ordre du blâme sur cette insignifiance? Cela me paraît tout à fait arbitraire.

Dans ma perspective, je dirai pour ma part, que cette signifiance n'existe que là où il $\mathrm{y}$ a rencontre, et qu'il ne suffit évidemment pas de croiser quelqu'un ou de se trouver à côté de lui dans un compartiment de chemin de fer pour qu'il y ait rencontre. Mais le terme et l'idée même de rencontre ne me semblent intervenir nulle part dans votre travail, et je voudrais savoir pourquoi.

En somme, ce qui me paraît très contestable dans votre position, c'est que, en posant le visage comme vous le faites, vous me paraissez aboutir à un formalisme du non-formel, du non formalisable.

Il faudrait examiner ici de très près la façon dont se lient pour nous visage et langage. En aucun sens il ne suffit qu'il y ait visage pour qu'il y ait aussi langage, même inarticulé, d'autant que pour vous, contrairement à Heidegger, le langage ne peut être conçu à partir d'un neutre, d'un es; c'est vraiment une relation, faut-il dire de personne à personne? Ici encore se pose un problème terminologique, vous n'usez pas du terme de personne et cela, me semble-t-il pour la raison que j'ai indiquée tout à l'heure.

Mais une question capitale se pose ici sur laquelle il ne me semble pas que vous ayez explicité suffisamment votre pensée: comment Dieu nous parle-t-il ? On peut se demander par instant si ce n'est pas ce langage et cette parole de Dieu qui est toujours présente, mais comme en retard. Mais cela vous ne le dites nulle part catégoriquement. 
Je conçois certes très bien que voulant nous maintenir sur le terrain purement philosophique, vous ayez jugé devoir, faut-il dire, refouler la référence au Dieu d'Israël. Mais je ne suis pas sûr que par là vous ne coupiez pas indûment votre pensée de ses racines, il me semble bien que ces racines sont bibliques, il y a là absolument rien qui personnellement me choque. En revanche, je me demande s'il n'est pas artificiel de pratiquer toutes sortes de coupures entre philosophie et religion.

Je présenterai encore l'objection sous une autre forme: je dirai que dès le moment où vous rejetez l'idée rationaliste d'un fondement de la valeur de l'autre (en tant que sujet) vous êtes en réalité dans l'obligation de chercher un fondement dans le Dieu vivant, le Dieu d'Abraham, d'Isaac et de Jacob. Encore une fois, je suis persuadé que c'est au fond votre pensée, mais je ne vois pas que vous puissiez justifier l'espèce de crainte ou de pudeur qui vous empêche de vous exprimer catégoriquement sur ce point. Si vous me répondez que Dieu ne peut pas être accueilli en Sorbonne, je vous dirai sans hésitation: tant pis pour la Sorbonne.

Il y a enfin une difficulté moins centrale peut-être, mais à mes yeux très sérieuse néanmoins que je dois évoquer. Elle porte sur l'usage que vous faites de la notion de fécondité. Sans doute faudrait-il à vrai dire, s'interroger d'abord sur votre phénoménologie de l'eros et sur le paradoxe qu'il y a d'avoir dissocié ce que vous dites de la caresse ou de l'érotisme des explications que vous avez fournies dans une section précédente sur la jouissance. Il y aurait là, je pense, matière à discussion serrée. Mais je suis plus préoccupé de ce que vous dites de la fécondité aux pages 244$247^{22}$. Nous retrouvons ici toute la difficulté que j'ai signalée à propos de l'infini. «L'être infini, dites-vous page 246, c'est-à-dire l'être toujours recommençant et qui ne saurait se passer de subjectivité, car il ne saurait sans elle recommencer - se produit sous les espèces de la fécondité». Je crains fort qu'en usant de ce terme vous n'aboutissiez à une très dangereuse naturalisation d'une pensée qui pourtant se place sur un plan tout autre. Il y a des moments, à vrai dire, où vous semblez identifier fécondité et maternité. Mais je dirai que la paternité est méta-biologique, ce qui n’est certainement pas le cas de la fécondité. Il y a paternité là où il y a adoption authentique; en d'autres termes l'essence de la paternité consiste à assumer, non pas du tout à procréer au sens biologique. Mais il faut dire aussi que vous simplifiez, je pense, de façon outrancière la relation entre père et fils. Le père ne se reconnaît pas nécessairement dans le fils. Le père peut sentir le fils comme totalement étranger et se demander avec angoisse ce

22. Totalité et Infini, quatrième section, « La fécondité ». 
que devient ou ce que signifie sa responsabilité. Nous sommes là dans une impasse. La vie sexuelle peut d'ailleurs en comporter d'autres, dont vous ne semblez pas vous soucier. Je pense par exemple aux problèmes de l'homosexualité auxquels vous ne faites même pas allusion. Et on aborderait là peut-être un autre type d'objections que votre beau livre peut soulever, ce que j'appellerais peut-être un abus dans la stylisation.

Vous avez, il est vrai, quelques très belles pages sur l'élément, sur ce que vous appelez le il y $a$, c'est-à-dire sur le ténébreux, mais laissez-vous voir suffisamment l'invasion possible du ténébreux au niveau de ce que j'appellerai pour ma part l'intersubjectivité? Je n'en ai pas tout à fait l'impression et ceci pourrait du reste m'amener à vous demander quelle est votre position métaphysique sur le mal. Il apparaît ici surtout comme meurtre mais comment le meurtre est-il possible? Par où suis-je exposé à le commettre? Qu'est-ce que la tentation? Y a-t-il un tentateur? 


\section{Annexe 3 \\ Compte rendu de Jacques Taminiaux sur Totalité et Infini}

\section{Louvain le 17 mai 1960}

Compte rendu d'un manuscrit de M. Emmanuel Levinas: «Totalité et Infini », de l'extériorité métaphysique. 383 pages dactylographiées.

Les êtres particuliers livrent-ils leur vérité dans un Tout où s'évanouit leur extériorité, et dont la guerre, ou la politique qui en est l'art, révèlent le concept? L'ultime événement de l'être se joue-t-il au contraire dans tout l'éclat de cette extériorité? Telle est la question à laquelle répond le travail de M. Levinas. Ce livre, qui oppose la philosophie, dominée par le concept de totalité, à l'eschatologie de la paix et de la justice dont la vision se consomme dans l'expérience morale, entend montrer que la totalité ne remplit pas la vraie mesure de l'être, que l'expérience morale atteste une relation avec un surplus toujours extérieur à la totalité et que le savoir lui-même a une situation qui ne peut plus se dire en termes de totalité: l'extériorité, la transcendance, ou l'infini se produisent dans le visage d'autrui.

Ce livre se présente donc comme une défense de la subjectivité, non pas au niveau de sa protestation égoïste ou de son angoisse devant la mort, mais comme fondée dans l'idée de l'infini, laquelle se produit dans la relation du Même avec l'Autre, relation par laquelle un être séparé, fixé dans son identité, s'avère contenir en soi ce qu'il ne peut contenir par la seule vertu de son identité. L'événement ultime de l'être n'est pas de l'ordre de la représentation, de l'évidence ou du dévoilement, il est l'extériorité radicale à laquelle aspire la métaphysique et qui meut la conscience à son niveau fondamental. De cet événement l'intentionnalité en tant qu'adéquation ne rend pas compte et l'auteur n'hésite pas à opposer l'idée cartésienne d'un débordement de l'infini sur le cogito à la tentative husserlienne de constituer l'infini par le cogito même.

De même, il reproche à Heidegger d'accorder priorité à l'être sur l'étant et par là de ramener l'Autre à l'identité neutre du Même, c'est-à-dire se subordonner l'éthique au savoir.

L'ouvrage est divisé en quatre sections. La première est intitulée Le Même et l'Autre. 
L'auteur y montre comment le désir métaphysique tend vers une altérité radicale, vers un pays qui n'est pas celui où nous sommes nés, vers un désiré qui ne comble pas le désir mais le creuse. En quoi le désir s’oppose au besoin, qui est manque allant vers sa satisfaction. Ce désir métaphysique est «transcendant», selon le terme de Jean Wahl, emprunté par l'auteur, il témoigne d'une absolue séparation entre le métaphysicien et la métaphysique. Comment, se demande l'auteur, le Moi qui s'identifie dans ses altérations, qui séjourne dans le monde, y étend son empire et s'y produit comme égoïsme, peut-il entrer en relation avec un Autre. Cette relation ne peut être représentation car l'Autre s'y dissoudrait dans le Même. Elle est langage. Le fait même d'être dans un discours, consiste à reconnaître à autrui un droit. Ici se brise la totalité et s'arrête le mouvement de la négativité et de la possession. Aborder autrui dans le discours, c'est accueillir son expression où il déborde l'idée qu'en emporterait une pensée, c'est recevoir de lui au-delà de la capacité du Moi, c'est avoir l'idée de l'infini. Il y a là une Sinngebung indépendante de son pouvoir.

Une telle relation qui est la métaphysique même, suppose la séparation du Même par rapport à l'Autre, mais non pas une séparation d'allure antithétique dont les termes s'appellent en se reposant et qu'embrasse un regard synoptique extérieur. La transcendance métaphysique exprimée par l'idée de l'infini doit se produire comme inintégrable, c'est-à-dire que la séparation du Moi à l'égard de l'Autre doit résulter d'un mouvement positif. Cette séparation se produit comme intériorité d'un psychisme se maintenant tout seul dans l'existence sans participer à l'Être dont il est séparé; cette séparation est athéisme. Elle n'est pas un moins par rapport à l'Infini, une déchéance de l'éternité, - ce qui supposerait une absorption dans l'Autre -, mais une satisfaction de soi qui cependant recherche l'Autre. Seul un être athée et heureux peut se rapporter à l'Autre et s'absoudre de cette relation. Cet athéisme conditionne une relation véritable avec un vrai Dieu corrélatif de la justice rendue aux hommes et se révélant dans la hauteur que manifeste autrui dans la relation irréductible du face-à-face.

L'auteur entend montrer comment ce commerce éthique, cette justice réclamée par autrui, est en étroite relation avec la vérité et la fonde. Le propre du savoir en effet est de se mettre en question, d'admettre une critique de la liberté dont l'essence est la spontanéité impérialiste du Même. Cette mise en question, cette critique commencent avec la conscience morale dans le rapport avec Autrui. Le dernier mot du savoir toujours à la recherche du fondement et d'une justification n'est pas le Pour Soi, ni le neutre de l'idée, de l'être ou du concept, mais l'avant-moi en présence d'autrui. C'est autrui qui offre le monde dans le langage, c'est lui qui est au principe du phénomène et du sens, c'est lui qui est à l'origine de la 
vérité comme Descartes l'avait bien vu lorsqu'il arrêtait le doute, une fois en possession de l'idée de l'infini, c'est-à-dire dans l'accueil à autrui. Le donné est le fait d'une parole; avoir un sens, c'est parler, enseigner ou être enseigné. Le rapport moral avec autrui, maître qui me juge, sous-tend la liberté de mon adhésion au vrai, lequel n'est pas dévoilement dans la solitude de la vision, mais parole.

La seconde section est intitulée Intériorité et économie. L'auteur y analyse les relations qui se produisent au sein du Même, et décrit l'intervalle de la séparation qui s'accomplit comme psychisme. La séparation est vie. Celle-ci est un acte se nourrissant de son activité, complaisance de soi dans les besoins. Vivre, c'est d'abord jouir de la vie? C'est par cette jouissance que s'accomplit la rupture de la totalité; être soi, c'est exister de telle manière qu'on soit déjà de l'être dans le bonheur. L'auteur oppose l'intentionnalité de cette jouissance à l'intentionnalité théorétique. Dans la représentation, le moi opposé au non-moi disparaît dans le Même, l'extériorité y est œuvre de pensée. Au contraire la jouissance tient à l'extériorité. Être corps, être besoin, c'est affirmer l'extériorité comme non-constituée. Par le corps le mouvement même de la constitution s'invertit, vivre de... c'est déterminer l'autre tout en étant déterminé par lui. C'est contester le privilège qu'on attribue à la conscience de prêter un sens à toute chose.

A partir de là l'auteur montre comment le séjour de l'homme dans le monde reste irréductible et antérieur à la connaissance de ce monde. L'intériorité de la jouissance est la séparation en soi. Être moi, athée, heureux chez moi, séparé, créé, ce sont là des synonymes. Cependant la jouissance est prise dans le contenu sans forme de l'élément et ne pas se complaire exclusivement en soi. La demeure est ce qui fonde ce recueillement. Par la maison s'instaure une extra-territorialité au sein des éléments, un délai dans la jouissance, qui rend possible le travail, la constitution des substances et l'éclairement de la nuit élémentale qui se mue en monde. Mais ce recueillement dans la demeure a une face positive; la douceur d'autrui, l'intimité de la femme, l'accueil. C'est donc par l'accueil, le don de ce que je possède à autrui qui me met en question, que je puis voir les choses en elles-mêmes et les représenter. C'est par lui que s'effectue la donation de sens qui est une dépossession originelle et non prolongement du mouvement de la jouissance et de la sensibilité.

La troisième section est intitulée Le visage et l'extériorité. L'auteur y montre la dimension de transcendance qu'introduit la relation avec autrui, visage et parole.

Le visage refuse d'être contenu dans le Même, et cependant autrui ne nie pas le moi, la mise en question qu'il opère instaure une relation éthique. Son 
expression, son épiphanie investit la liberté et suscite sa responsabilité. Avant toute intentionnalité constituante, fut-elle corporelle, et contestant la liberté constituante, se trouve la relation première de l'obligation. La première signification est apportée par le visage. Il est l'évidence qui rend possible l'évidence, le premier enseignement et la première raison, le fondement de l'objectivité qui est entrée de la chose dans la sphère de l'autre. Cette relation éthique est ouverte à l'humanité, à la vraie raison, et au temps, surplus et nouveauté inépuisable de l'infini. La raison commence dans l'acte positif qui consiste pour chaque interlocuteur à donner le monde à l'autre et à justifier devant l'autre de sa liberté, par la bonté.

Dans la quatrième section intitulée $A u$-delà du visage, l'auteur explore un plan qui à la fois suppose et transcende l'épiphanie d'Autrui dans le visage. La violence de la mort réduit au silence la subjectivité et semble accepter de se taire révoltée par la violence de la raison impersonnelle, tyrannie, histoire et Etat, elle peut renoncer d'elle-même à soi sans violence. Cette renonciation est l'amour. Le plan de l'amour et de la fécondité est celui où le moi se porte au-delà de la mort et se relève aussi de son retour à soi. L'auteur après avoir esquissé une phénoménologie de l'Eros montre comment la fécondité est la transcendance du moi, par laquelle il se dépouille de son égoïsme sans cependant se dissoudre dans le collectif.

En conclusion enfin, l'auteur rappelle les thèmes principaux de son ouvrage: la trace de l'être est la relation sociale, l'idée de l'infini, la présence d'un contenu dans un contenant dépassant la capacité du contenant. L'être est extériorité, le fini n'est pas une déchéance de l'infini, le multiple n'est pas une chute de l'Un. Contre la philosophie du neutre dont il voit la plus récente expression dans la pensée de Heidegger, l'auteur défend un Logos qui est verbe d'une personne et exigence éthique. Au-delà de l'être de l'ontologie il y a le Bien, l'auteur à sa manière souscrit à cette pensée platonicienne. 